\title{
GENERACIÓN DE ACELEROGRAMAS SINTÉTICOS MEDIANTE EL USO DE WAVELETS, ORIENTADOS A APLICACIONES GEOTÉCNICO- ESTRUCTURALES
}

\author{
Francisco Alonso Flores-López ${ }^{(1)}$ y Juan Carlos Ayes-Zamudio ${ }^{(2)}$
}

\begin{abstract}
RESUMEN
En el presente trabajo se expone una secuencia de análisis orientada a la generación de acelerogramas sintéticos mediante el empleo de wavelets. Se presentan criterios generales, la aplicabilidad de éstos así como conclusiones de utilidad práctica para generar acelerogramas sintéticos para su empleo en análisis geotécnicos o estructurales y de interacción suelo-estructura. Se generan acelerogramas sintéticos para diversos tipos de suelos (suelos rígidos y suelos blandos de la Ciudad de México y Caleta de Campos, Michoacán) y para varios espectros objetivo como lo son: espectros de diseño normativos, espectros de peligro uniforme y espectros derivados de envolventes de registros sísmicos. Se observa la estabilidad del método para generar acelerogramas sintéticos asociados a cualquier espectro objetivo y se muestra que, empleando este método para ajustar señales reales, las modificaciones que sufren los espectros de Fourier así como las intensidades de Arias son mínimas. Con los ejemplos mostrados en este trabajo se concluye que la generación de los acelerogramas sintéticos empleando wavelets es muy estable y partiendo de los mismos sismos semilla se pueden generar acelerogramas sintéticos para distintos espectros objetivos.
\end{abstract}

Palabras clave: ajuste espectral; registros sísmicos sintéticos; wavelet

\section{GENERATION OF SYNTHETIC ACCELEROGRAMS USING WAVELETS, ORIENTED TO GEOTECHNICAL-STRUCTURAL APPLICATIONS}

\begin{abstract}
In this work, a procedure to generate synthetic accelerograms using wavelets is presented. General criteria and its applicability are presented, as well as conclusions of practical use to generate synthetic accelerograms for use in geotechnical or structural analysis and soil-structure interaction. Synthetic accelerograms are generated for different types of soils (rigid soils and soft soils of Mexico City and Caleta de Campos, Michoacán) and for several objective spectra as they are: normative design spectra, uniform hazard spectra and spectra derived of envelopes of seismic records. The stability of the method to generate synthetic accelerograms associated to any objective spectrum is shown, in addition to demonstrating that the modifications suffered by the Fourier spectra as well as the Arias
\end{abstract}

\footnotetext{
Artículo recibido el 8 de marzo de 2017 y aprobado para su publicación el 3 de junio de 2018. Se aceptarán comentarios y/o discusiones hasta cinco meses después de su publicación.

(1) Instituto Mexicano del Petróleo, Coordinación de Geotecnia Marina, Eje Central Lázaro Cárdenas Norte 152, San Bartolo Atepehuacán, Ciudad de México. C.P. 07730, faflores@imp.mx

(2) Consultor, Juan de Dios Arias 1, Vista Alegre, Ciudad de México. C.P. 06860, jcaz15@ hotmail.com
} 
intensities when this method is used to adjust real signals are minimal. With the examples shown in this work, it is concluded that the generation of the accelerograms employing wavelets is very stable and starting from the same seismic records it can generate synthetic accelerograms for different objective spectra.

Keywords: spectral matching; synthetic seismic records; wavelets

\section{INTRODUCCIÓN}

La simulación dinámica no lineal de un sistema geotécnico, estructural o mixto, es un problema complejo y el uso de espectros elásticos no se considera como un método adecuado para generar análisis a detalle. El concepto de ajuste espectral es el modificar una historia de tiempo semilla de tal manera que su espectro se ajuste de manera cercana al espectro objetivo (espectro de diseño, espectro de peligro uniforme, etc.) en un amplio rango de periodos; modificando lo menos posible parámetros relacionados a la amplitud, contenido frecuencial y duración (e.g. aceleración máxima del terreno, espectro de respuesta, duración significante, intensidad Arias, etc.).

Una alternativa de ajuste espectral, respecto al escalado lineal y al ajuste en el dominio de la frecuencia es la adición de ondas, en este caso wavelets, la cual es una función matemática que está localizada tanto en el dominio del tiempo como en el de la frecuencia, esta habilidad de expresar señales en ambos dominios es particularmente útil para el análisis de señales no estacionarias como las sísmicas. Un procedimiento aceptado es el uso de historias de tiempo o HDT (término que engloba a las historias de aceleraciones HDT-a, de velocidades HDT-v o de desplazamientos HDT-d) que presenten un preprocesamiento adecuado (Ayes y Flores, 2015; Ayes, 2016) y la cual se encuentre ajustado a un espectro de respuesta que represente las acciones sísmicas de un determinado depósito de suelo. La acción de la fuente sísmica se incluye al seleccionar los registros sísmicos medidos en una o varias estaciones sismológicas para diferentes fuentes sísmicas y éstas hacerlas compatibles con un espectro de respuesta objetivo. Como preámbulo a cualquier diseño anti-sísmico, tanto desde la perspectiva estructural como geotécnica, siempre es necesario determinar con antelación las características de los movimientos sísmicos esperados en la zona de interés; de tal manera que la caracterización geosísmica es una de las primeras actividades a realizarse antes de cualquier intento de análisis numérico, siendo posible realizar ésta de manera determinista o probabilista. Desde una perspectiva ingenieril y con el fin de utilizar historias de tiempo en análisis orientados a la definición de los efectos de un sismo en una determinada estructura en el dominio temporal, es importante, estimar el nivel de ruido existente en cada HDT, así como el nivel o grado con el cual éste afecta a los distintos parámetros derivados de su interpretación (e.g. ordenadas espectrales, aceleración máxima del terreno (PGA), velocidad máxima del terreno (PGV), desplazamiento máximo del terreno (PGD)). La finalidad del ajuste espectral es el de identificar o crear uno o más movimientos con amplitudes, contenido de frecuencia y duración, consistentes con el nivel de riesgo sísmico analizado; por ejemplo ASCE/SEI 7-05 (2006) requiere que los movimientos sísmicos registrados sean escalados de tal manera que el espectro de respuesta promedio en términos de aceleraciones, se aproxime al espectro de respuesta en término de aceleraciones en roca del sismo máximo considerado (MCE, Maximum Considered Earthquake) en el rango de periodos de interés. Es interesante mencionar, que el procedimiento de ajuste espectral está ganando aceptación dentro del diseño estructural, esto debido a que el diseño sismo-resistente se está alejando del enfoque espectral reglamentario y enfocándose a los procedimientos espectrales desarrollados como parte de los análisis de respuesta de sitio. El manual de CFE (2015) indica que los parámetros espectrales para estructuras del grupo A+ y A1 se obtendrán a partir de análisis de respuesta de sitio, donde se calcularán acelerogramas sintéticos en la superficie del terreno. FEMA (2012), incluye análisis en el dominio del tiempo (análisis directos) para evaluar los efectos de interacción dinámica suelo-cimentaciónestructura. En los análisis paso a paso, es indispensable el contar con un movimiento de entrada (artificial, 
registrado, ajustado, etc.), esto porque en la mayoría de los casos, no es posible obtener registros sísmicos en un sitio determinado y aunque así fuera, es difícil esperar que un sismo futuro en una zona en particular pueda generar el mismo nivel de energía-aceleración-movimiento, por lo cual, normalmente se requiere generar acelerogramas sintéticos ajustados a espectros de referencia (e.g. reglamentarios, de peligro uniforme, etc.). Con esto surge la necesidad de generar señales que representen el ambiente sísmico máximo creíble (e.g. amplitud, duración, etc.) modificando la respuesta espectral de movimientos registrados y hacerlos compatibles con la respuesta espectral máxima reglamentaria. El objetivo de este trabajo es exponer procedimientos orientados a la generación de acelerogramas sintéticos mediante el empleo de wavelets y presentar criterios generales, aplicabilidad de estos, comentarios a los procedimientos, así como conclusiones de utilidad práctica.

\section{PROCEDIMIENTOS EXISTENTES PARA REALIZAR AJUSTES ESPECTRALES}

Actualmente existe una amplia variedad de procedimientos con el fin de modificar los acelerogramas semilla para ajustarlos a un espectro de referencia u objetivo. A continuación, se presenta una breve descripción de algunos procedimientos, sus ventajas y limitaciones.

\section{Escalado lineal}

En este procedimiento, el acelerograma en su totalidad es escalado de manera directa "hacia arriba o hacia abajo" por un factor constante, típicamente este factor de escalado es seleccionado para lograr un ajuste al PGA o un ajuste en la zona cercana al periodo fundamental de la estructura estudiada. Su finalidad es alterar las amplitudes de una señal tal que su respuesta espectral coincida con algún parámetro (e.g. PGA) o generar el ajuste espectral en la zona cercana al periodo fundamental de la estructura analizada. Este procedimiento es sencillo de generar, además de que el contenido de frecuencias y la fase original del acelerograma se conserva; no existen reglas o procedimiento establecido, pero es recomendado no utilizar factores muy altos (e.g. mayores que $2-3$ ), debido a que el resultado puede ser no realista en términos de amplitudes relativas, duración y para sismos de magnitud importantes un contenido de frecuencias diferentes a la historia original. Siempre es necesario examinar de manera crítica el espectro escalado, con el fin de determinar si existe una sobre-generación o deficiencia severa para periodos diferentes al periodo objetivo. En las figuras 1 y 2, se realiza un escalado lineal hasta el PGA del espectro de referencia para dos estaciones acelerográficas ubicadas en la Ciudad de México (CU, zona I y SCT, zona III); es posible observar que el escalado no necesariamente permite a la HDT escalada presentar valores espectrales similares al espectro de referencia (estación CU) y en cambio puede producir un exceso de aceleraciones espectrales para ciertos periodos (estación SCT) en periodos de sumo interés ingenieril (1.0 - $3.0 \mathrm{~s})$. Aunque por lo anteriormente expuesto, pareciera que su utilidad práctica es limitada, su uso es aconsejable cuando se realizan ajustes espectrales en el dominio del tiempo, lo que en general permite evitar hacer grandes modificaciones a la HDT y modificar en exceso su contenido de frecuencia.

\section{Escalado en el dominio de la frecuencia}

Bommer y Acevedo (2008) realizaron un trabajo acerca del uso de registros sísmicos reales como datos de entrada en análisis dinámicos y comentan que los acelerogramas sintéticos puede ser un complemento al uso de registros sísmicos resaltando que cada uno tendrá sus propios méritos para diferentes aplicaciones, siendo los acelerogramas sintéticos una opción atractiva para escenarios en los cuales se pretenda hacerlos compatibles con espectros de diseño objetivos. Los primeros métodos para realizar ajustes espectrales fueron diseñados para trabajar en el dominio de la frecuencia, estos métodos se basan en ajustar el espectro de amplitud de Fourier (FAS) basado en la relación existente entre el espectro de respuesta objetivo y el espectro de respuesta de la señal original. Un ejemplo de ajuste espectral de este tipo es el 


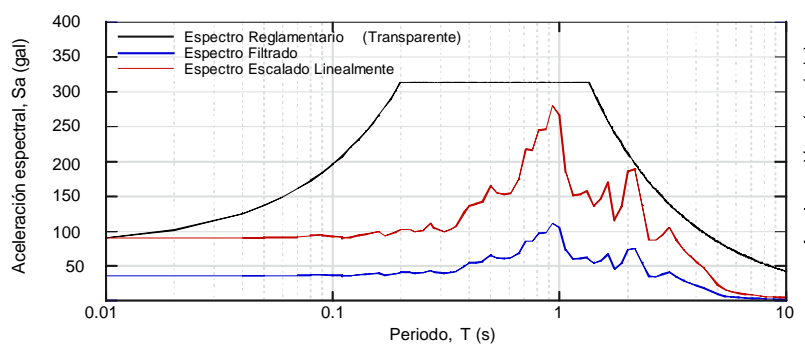

(a)

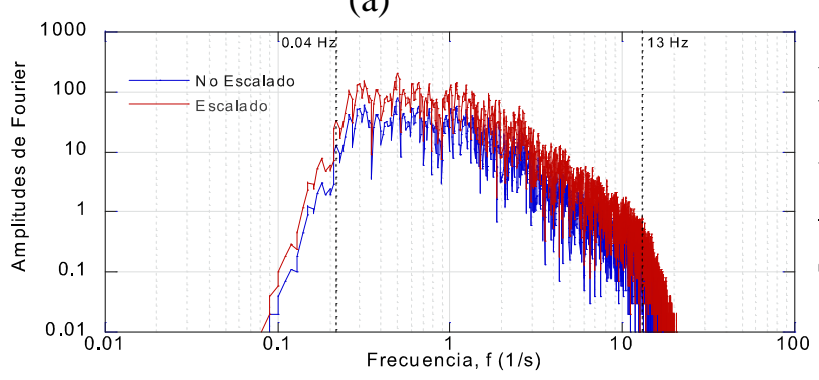

(c)

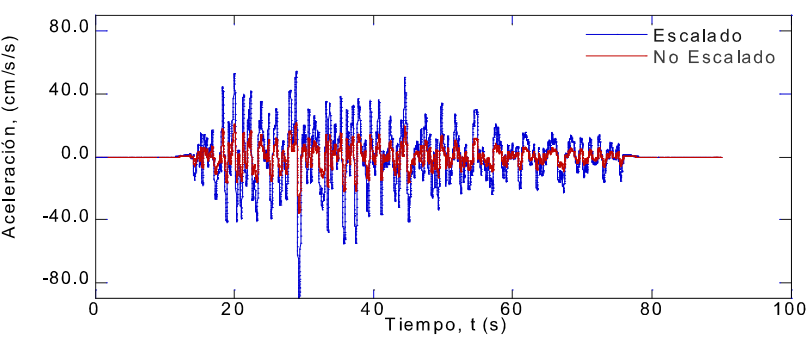

(b)

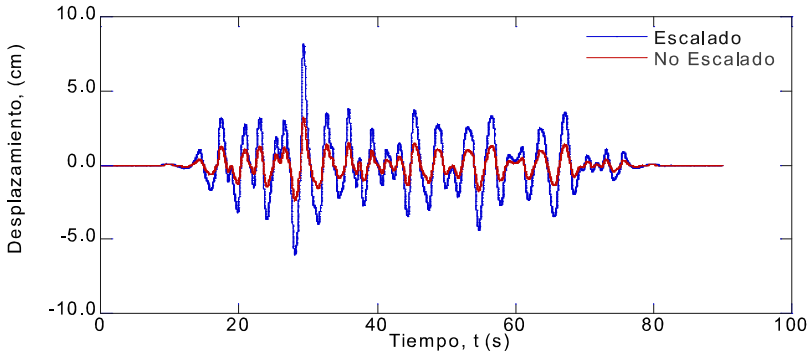

(d)

Figura 1. Comparación del escalado lineal, (a) Espectros de aceleraciones, (b) HDT-a en aceleraciones, (c) Espectros de amplitudes de Fourier y (d) HDT-d en desplazamientos (estación CU, 19/09/1985)

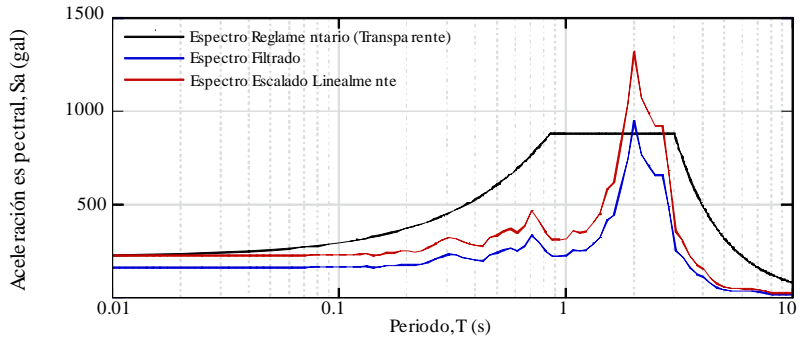

(a)

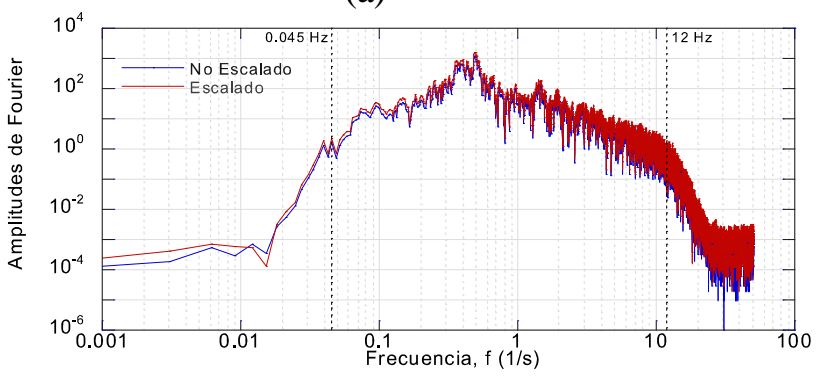

(c)

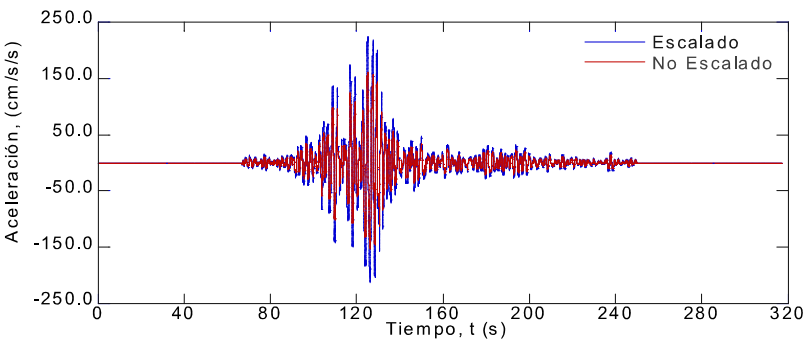

(b)

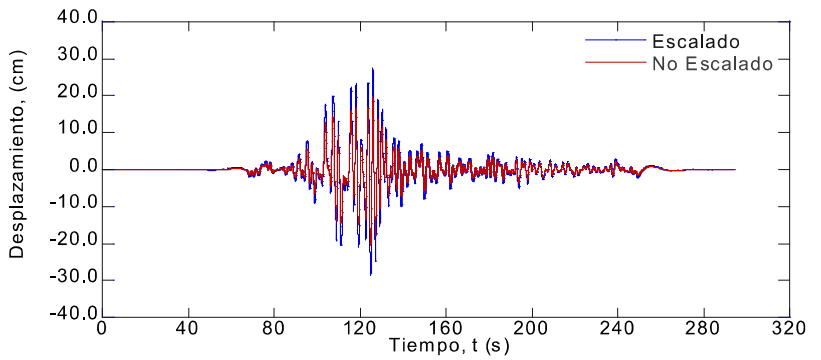

(d)

Figura 2. Comparación del escalado lineal, (a) Espectros de aceleraciones, (b) HDT-a en aceleraciones, (c)

Espectros de amplitudes de Fourier y (d) HDT-d en desplazamientos (estación SCT, 19/09/1985)

trabajo desarrollado por Gasparini y Vanmarcke (1979) mediante el uso del programa SIMQKE, cuyo procedimiento se basa en generar una función de densidad espectral a partir del espectro de respuesta objetivo, para posteriormente derivar señales sinusoidales con amplitudes aleatorias, finalmente tales señales son adicionadas y mediante un procedimiento iterativo es posible mejorar el ajuste obtenido. Sabetta y Pugliese (1996), proponen un método empírico para simular historias de tiempo basado en la sumatoria de series de Fourier con fases aleatorias y coeficientes que dependen del tiempo. Softwares basados en esquemas en el dominio de la frecuencia fueron desarrollados por diferentes autores (e.g. RASCAL, Silva y Lee, 1987; SYNTH, Naumoski, 2001). La principal ventaja del escalado en el dominio de la frecuencia, 
es que se puede obtener ajustes completos a un espectro elástico de diseño, ya que en múltiples ocasiones éste, es la única información disponible para el ingeniero; sin embargo, tal enfoque genera un excesivo número de ciclos en la HDT y por lo tanto un gran contenido de alta energía; de lo anterior, aunque el procedimiento es directo, no mantiene el carácter no estacionario de la HDT y genera señales que no se parecen a una señal sísmica empírica. En la figura 3 se muestra la comparación entre la HDT original de la estación CU y la generada mediante el programa para RSCHT (en su versión más reciente TARSCHT, creado para generar acelerogramas artificiales), la cual está ajustada a un espectro reglamentario, de ahí las diferencias espectrales.

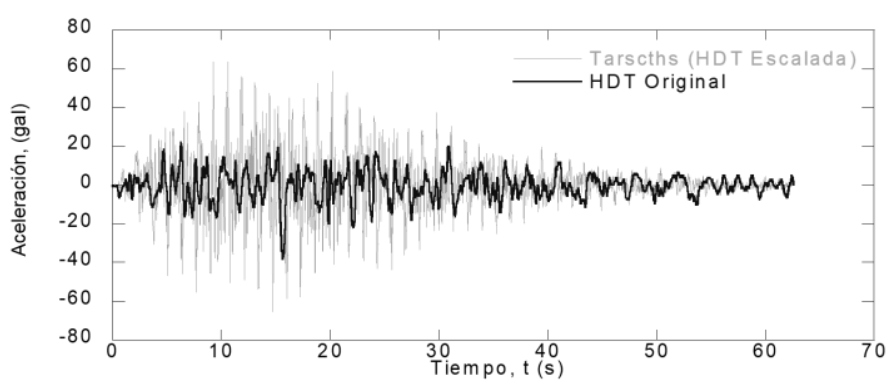

(a)

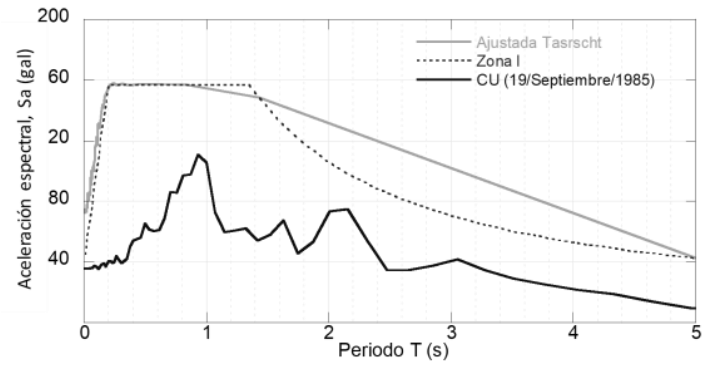

(b)

Figura 3. Comparación entre la HDT original de la estación CU y la generada mediante TASRSCHT

Aunque existen diversos enfoques en el ajuste espectral, incluyendo el uso de la teoría de vibración aleatoria (Random Vibratory Theory, RVT) (Kottke et al., 2013), y tomando en cuenta el estado actual de la práctica, consideramos que los ingenieros de la práctica cuentan con una amplia variedad de técnicas para incluir los movimientos sísmicos en el diseño de estructuras, y éstas se deben seleccionar dependiendo de la información con la que cuente del sitio particular de estudio que este analizando. Para reducir la dispersión de los movimientos individuales algunos investigadores proponen métodos que ajustan la respuesta espectral individual mediante el ajuste de la amplitud de Fourier; esto es útil debido a que genera acelerogramas que están basados en movimientos reales y con un alto ajuste al espectro de referencia. Desafortunadamente, el ajuste del espectro de Fourier corrompe la velocidad y el desplazamiento de la HDT y puede resultar en movimientos con un alto y no realista contenido energético. Por otro lado, Cecini y Palmeri (2015) consideran que la generación de acelerogramas a partir de métodos estocásticos donde no se considera la génesis sísmica real y las señales del movimiento del suelo se manejan matemáticamente como un proceso aleatorio, no es ideal para su uso en algunas aplicaciones ingenieriles, especialmente en sistemas geotécnicos donde la respuesta del suelo está altamente influenciada por las deformaciones angulares inducidas durante el movimiento sísmico y en análisis donde el contenido de energía tiene un papel importante como el caso del fenómeno de licuación de arenas.

Por otra parte, existen métodos basados en modelos físicos mediante funciones de Green empíricas [e.g. Hartzell (1978), Ordaz et al. (1995) y Kohrs-Sansorry et al. (2005)] que emplea pequeños sismos (funciones de Green) que se generan en distintos instantes de tiempo en la zona de ruptura o de deslizamiento, con la finalidad de generar el movimiento del terreno. Estos métodos tienen la ventaja que se basan en modelos físicos. Sin embargo, esta técnica requiere como dato de entrada conocer registros sísmicos en el sitio de interés, al igual que el método basado en wavelets indicado en este trabajo.

\section{Escalado en el dominio del tiempo}

Para reducir el número de análisis requeridos para obtener estimados estables de la respuesta de estructuras no lineales o críticas, es deseable reducir la variabilidad del movimiento sísmico de entrada; lo anterior puede ser alcanzado mediante el uso de HDT que presenten una respuesta espectral cercana al 
espectro elástico de diseño u objetivo. Una alternativa de ajuste espectral, respecto al escalado lineal y al ajuste en el dominio de la frecuencia es la adición de ondas, en este caso wavelets, la cual es una función matemática que está localizada tanto en el dominio del tiempo como en el de la frecuencia, esta habilidad de expresar señales en ambos dominios es particularmente útil para el análisis de señales no estacionarias como la de los movimientos sísmicos. Adicionalmente esta función presenta una duración finita con un promedio igual a cero, su amplitud típicamente inicia en cero, se incrementa y posteriormente regresa a cero; en otras palabras, es una función harmónica o repetitiva que está concentrada en una duración finita, las wavelets pueden tener distintas formas funcionales (figura 4). El ajuste de HDT mediante wavelets a HDT presenta las mismas ventajas que el ajuste del espectro de Fourier, pero conlleva a una adecuación o corrección más focalizada, todo lo anterior en el dominio del tiempo, por lo que el procedimiento introduce menos energía al movimiento sísmico (figura 5).

\section{AJUSTE ESPECTRAL MEDIANTE WAVELET}

El desarrollo del procedimiento de ajuste espectral en el dominio del tiempo mediante el uso de wavelets inició formalmente mediante el algoritmo propuesto por (Lilhanand y Tseng, 1988), aunque basado en el trabajo de Kaul (1978), tal método generalmente produce buenos resultados en relación con el ajuste espectral de la señal modificada cuando se compara su espectro de respuesta con el espectro objetivo, sin embargo, se presentan dos principales problemas: 1) La wavelet utilizada corrompe la velocidad y el desplazamiento de la historia de tiempos de los acelerogramas, de tal manera que es necesario realizar corrección de línea base (CLB) después de cada ajuste, lo cual puede parcialmente deshacer el ajuste espectral realizado, 2) el método no siempre es estable y diverge si se utilizan periodos espaciados muy cercanos uno al otro.

La metodología propuesta por (Lilhanand y Tseng, 1988), utiliza funciones wavelets, pero no está basada en la teoría convencional de wavelets (Hancock et al., 2006), la principal diferencia radica en que se usa la respuesta de sistemas elásticos de un grado de libertad para analizar las HDT, más que en las transformadas wavelets.

Con base a una amplia variedad de análisis paramétricos empleando distintas HDT, los autores proponen una secuencia de análisis basada en gran parte en el trabajo pionero de (Lilhanand y Tseng, 1988), pero integrando sugerencias relacionadas a la selección y empleo de filtros y su efecto en las HDT sintéticas.

En general, la secuencia de análisis es posible resumirla en tres etapas: (1) Cálculo de la respuesta de un sistema elástico de un grado de libertad bajo la acción de una HDT para cada periodo y para el nivel de amortiguamiento a ser ajustado, (2) Comparación de la respuesta espectral máxima (respuesta pico) de cada sistema con la amplitud espectral objetivo y determina la diferencia existente (mismatch) y (3) Adición de wavelets a la HDT con las amplitudes y fases apropiadas, de tal manera que el pico de cada respuesta espectral coincida con la amplitud objetivo. La principal suposición del método es que asume que la respuesta pico del oscilador elástico SDOF no cambia como resultado de los ajustes inducidos por las wavelets.

\section{GENERACIÓN DE ACELEROGRAMAS SINTÉTICOS MEDIANTE RSPMATCH}

El programa más utilizado para la generación de ajustes espectrales en el dominio del tiempo en la ingeniería práctica a nivel mundial es el reconocido RSPMATCH (ReSPonse MATCH) del cual, al día de hoy existen varias versiones, v.1992, v.2005 y v.2009 (Al Atik y Abrahamson, 2010), sus diferencias radican esencialmente en el tipo de wavelet utilizada. 

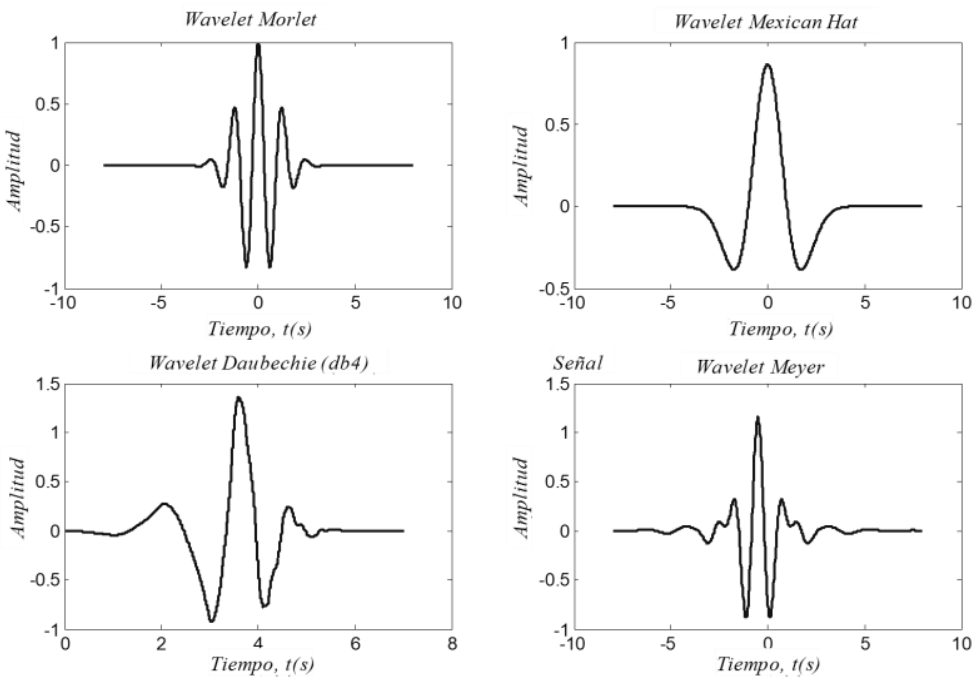

Figura 4. Tipos de wavelet básicas

(a)

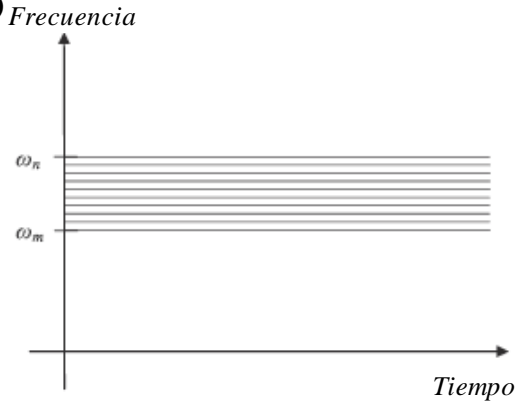

(c)

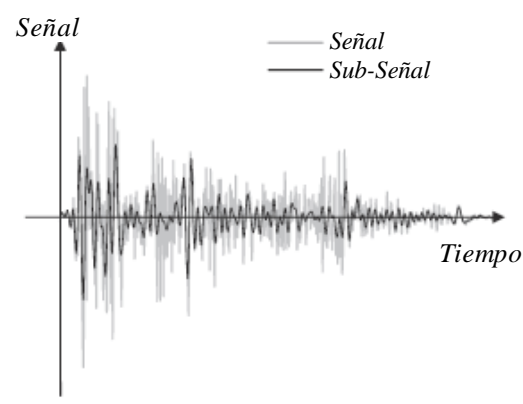

(b)

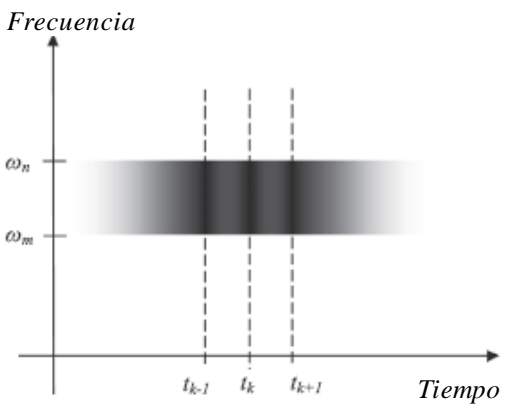

(d)

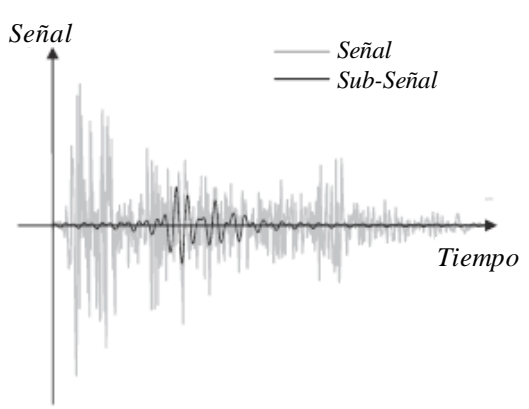

Figura 5. Extracción de sub-señales mediante transformada de Fourier (a y c) versus transformada wavelet armónica (b y d) (Tomado de Cecini y Palmeri, 2015)

La primera versión (v.1992) se basa en dos wavelets: Reverse Acceleration Impulse Response (Lilhanand y Tseng, 1988) y Tapered Cosine (Abrahamson N., 1992), este algoritmo preserva el carácter no estacionario del movimiento de referencia y asegura la estabilidad y eficiencia de la solución numérica; sin embargo, las funciones de ajuste no integran a cero tanto en velocidades como desplazamientos (figura 6a) generando así un desfase en las respectivas HDT; por lo tanto, para su aplicación práctica se requiere la aplicación de una CLB a la HDT-a en cada pasada, lo cual resulta tardado y laborioso.

La segunda versión (v.2005) (figura 6b), mejoró el algoritmo mediante la función de ajuste en la cual se incluyó la CLB en su forma funcional, denominada Improved Tapered Cosine, debido a lo anterior, ya 
no era posible tener una solución analítica y por lo tanto se comprometió la velocidad y eficiencia del método debido a la necesidad de integración numérica.

Finalmente, la tercera versión (v.2009), propone una nueva función de ajuste de igual nombre al anterior, la cual permite el uso de una solución analítica y cuya forma funcional integra directamente a cero tanto en velocidades como desplazamientos (figura 6c), esto sin la necesidad de realizar una CLB.

Una de las características principales en el método propuesto, es la elección de la función o wavelet de ajuste, tal elección debe basarse en los resultados de compatibilidad, así como en la estabilidad numérica.
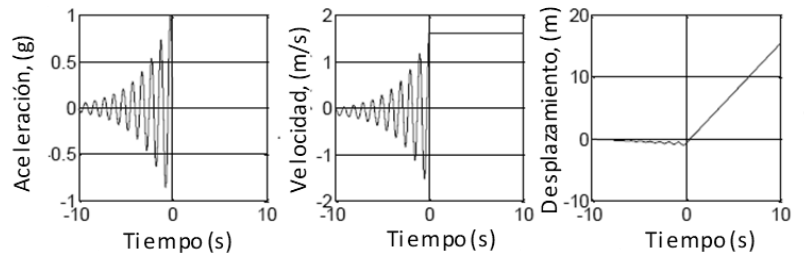

a. Lilhanand y Tseng, 1988; Abrahamson N., 1992

$f_{j}(t)=h_{j}\left(t_{j}-t\right)=\frac{\cdots}{\sqrt{1-\beta_{j}^{2}}} \exp \left(-\omega_{j} \beta_{j}\left(t_{j}-t\right)\right)\left[\left(2 \beta_{j}^{2}-1\right) \sin \left(\omega_{i}^{\prime}\left(t_{j}-t\right)\right)\right.$

$\left.-2 \beta_{j} \sqrt{1-\beta_{j}^{2}} \cos \left(\omega_{j}^{\prime}\left(t_{j}-t\right)\right)\right]$,
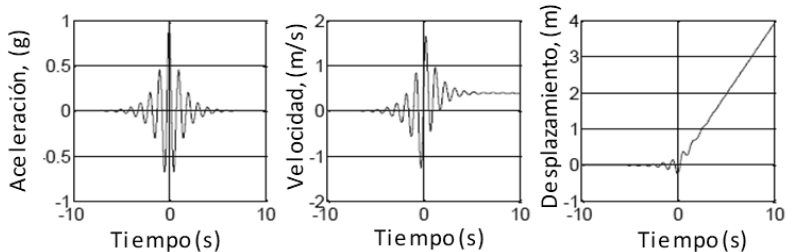

b. Tapered Cosine (Hancock et al., 2006)

$$
\begin{gathered}
f_{j}(t)=\cos \left[\omega_{j}^{\prime}\left(t-t_{j}+\Delta t_{j}\right)\right] \exp \left[-\left|t-t_{j}+\Delta t_{j}\right| \alpha_{j}\right], \\
\Delta t_{j}=\frac{\tan ^{-1}\left[\frac{\sqrt{1-\beta_{j}^{2}}}{\beta_{j}}\right]}{\omega_{j}^{\prime}} .
\end{gathered}
$$
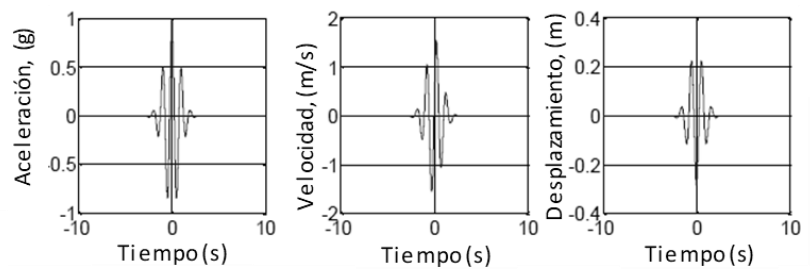

\section{c. Improved Tapered Cosine (Al Atik L. \&} Abrahamson N., (2010)

$$
\begin{gathered}
f_{j}(t)=\cos \left[\omega_{j}^{\prime}\left(t-t_{j}+\Delta t_{j}\right)\right] \exp \left[-\left(\frac{t-t_{j}+\Delta t_{j}}{\gamma_{j}}\right)^{2}\right], \\
\gamma(f)=1.178 f^{-0.93}
\end{gathered}
$$

Figura 6. Tipos de wavelets utilizados en el programa RSPMatch09, a) Reverse Acceleration Impulse Response b) Tapered Cosine y c) Improved Tapered Cosine, (modificada de Al Atik y Abrahamson, 2010)

Por otra parte, en la literatura existen varias investigaciones recientes relacionadas con el uso de la transformada wavelet para generar acelerogramas sintéticos. Giaralis y Spanos (2009) emplearon un esquema estocástico para simular historias de tiempo no estacionarias compatibles con espectros de desplazamientos basados en un procedimiento iterativo empleando wavelets armónicas. Cecini y Palmeri (2015) desarrollaron un procedimiento iterativo basado en la Transformada Wavelet Armónica para generar ajustes espectrales por medio correcciones a los registros sísmicos, tanto en el tiempo como en la frecuencia. Zhang et al., (2015) propusieron un método para realizar ajustes espectrales en el dominio del tiempo empleado lo que denominaron Corrective displacement wavelet, mejorando así la estabilidad, eficiencia y rapidez de los métodos basados en la transformada Wavelet. Estas publicaciones son parte del estado del arte actual, pero que todavía no se incluyen en los análisis sísmicos en la ingeniería práctica.

\section{ACELEROGRAMAS SINTÉTICOS ASOCIADOS A DIVERSOS ESPECTROS OBJETIVO}

Debido a la disponibilidad práctica, la generación de acelerogramas sintéticos se realizó con el programa RSPMATCH v.2009 y empleando el modelo propuesto por Atik y Abrahamson (2010). Para generar los acelerogramas sintéticos es necesario contar con: a) Espectro objetivo (figura 7) típicamente un 
espectro reglamentario o un espectro obtenido de un análisis de riesgo sísmico (espectro de peligro sísmico, EPU); b) Rango de periodos de interés y c) Historias de tiempos (HDT) compatibles (figura 8), es decir, HDT modificadas adecuadamente con el fin de permitir la obtención de velocidades y desplazamientos físicamente admisibles mediante la integración directa de la HDT-a (sismo semilla).

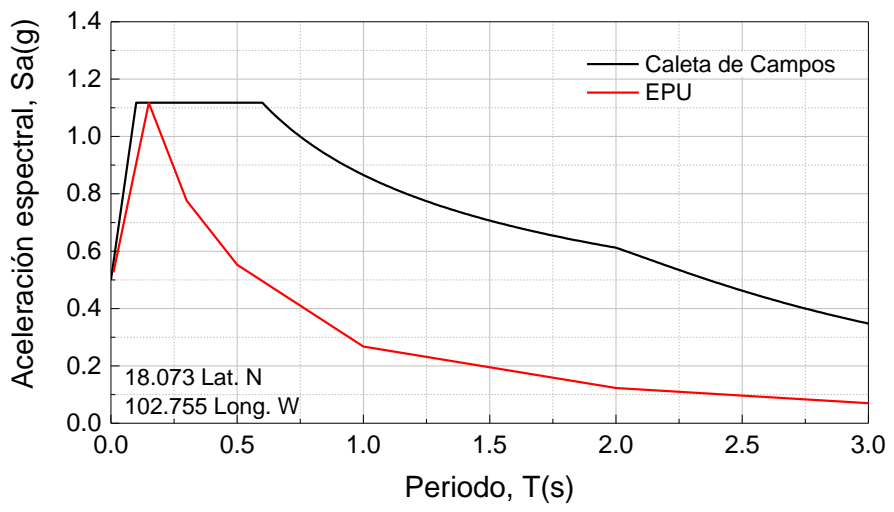

Figura 7. Ejemplo de posibles espectros objetivos (espectros de Caleta de Campos, Michoacán) mediante PRODISIS v4.1 (CFE, 2015)
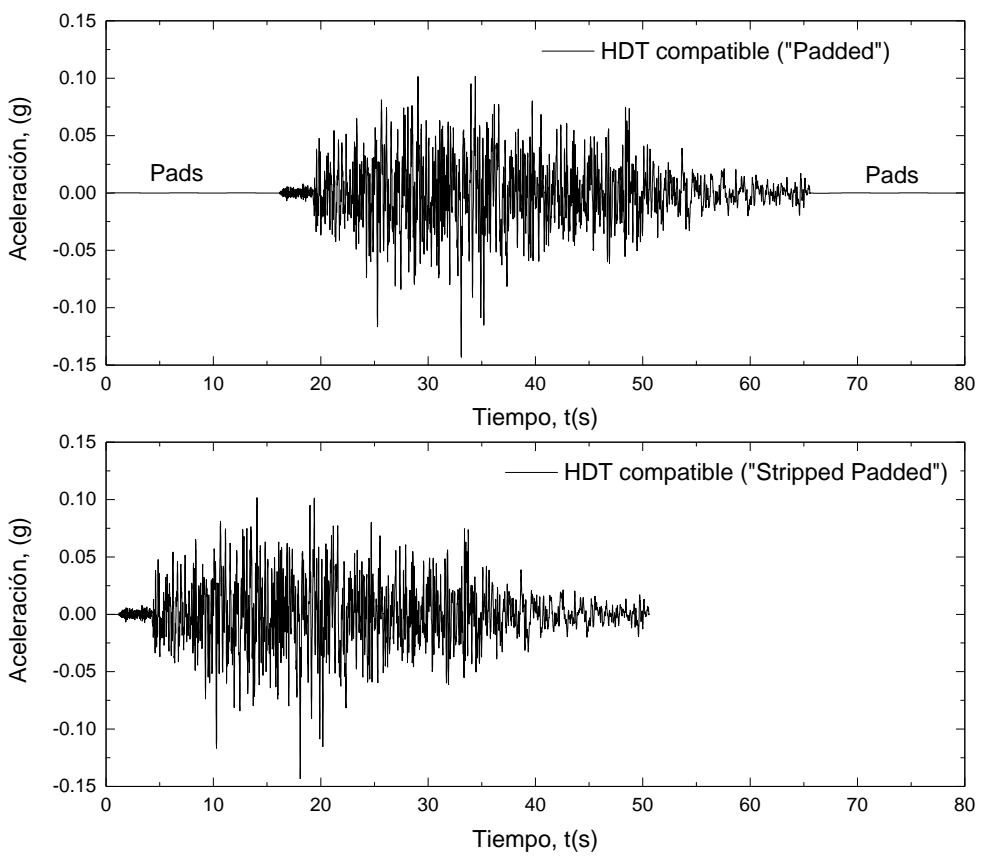

Figura 8. HDT compatibles en versión "Padded" y "Stripped padded" correspondiente a la estación "Caleta de Campos", 19/09/1985 (0.20-40 Hz)

Como resultado de la aplicación del modelo de Atik y Abrahamson (2010), se tiene una HDT ajustada en todo el rango de amplitudes con el espectro objetivo, es decir, sufrió de modificaciones en su amplitud (éstas últimas son función de la diferencia espectral entre el espectro original y el espectro objetivo), sin modificar su fase estacionaria, ni su longitud; sin embargo, tal HDT no es compatible, por lo que para obtener nuevamente tal compatibilidad entre HDT-a, HDT-v e HDT-d, se genera un nuevo análisis de tratamiento de la señal mediante filtrado (Ayes J. y Flores A., 2015), pero con frecuencias de corte menores 
o mayores a las frecuencias antes utilizadas, de tal manera que no se modifique su respuesta, pero si se adicione el número necesario de pads a la HDT (se entiende como pad a la adición de líneas de datos con una amplitud nula o muy cercana a cero, tales adiciones se hacen al inicio y al final del registro sísmico; tal longitud depende de la frecuencia y el orden del filtro, ver Converse y Brady, 1992).

Una historia de tiempos, presentada con la totalidad de pads adicionados después del procedimiento de filtrado se le denomina padded, en caso contrario una sin la presencia de la totalidad de pads, se le denomina stripped padded.

Se recomienda que las frecuencias mayores empleadas en el análisis para obtener nuevamente la compatibilidad entre las distintas historias, sean mayores a la frecuencia de Nyquist, la cual se define como: la máxima frecuencia a la cual las características de una señal pueden ser determinadas y es igual a:

$f_{\text {Nyquist }}=\frac{1}{2 \Delta t} ;\left(\right.$ e.g. $\left.\Delta t=0.01 s \therefore f_{\text {Nyquist }}=50 H z\right)$

donde $\Delta t$ es el intervalo de tiempo en una HDT. Si se aplica un filtro low-pass a frecuencias mayores que la frecuencia de Nyquist, tal filtro no tendrá efecto alguno en el registro analizado.

En la figura 9a se muestra el ajuste espectral realizado para dos señales, una registrada en suelo rígido y otra señal medida en suelo blando. Los espectros objetivos empleados corresponden a los espectros de diseño de las NTC, 2004 para zona de lomas y zona de lago, respectivamente. En la figura 9b se muestra la historia de tiempos semilla y modificada. La historia de tiempos modificada es el sismo sintético generado mediante el procedimiento descrito, el cual permite obtener una HDT con características propias del registro empírico. La figura 9c presenta la gráfica de amplitudes adicionadas a la HDT durante el proceso del ajuste espectral. Puede verse en estas figuras que el ajuste espectral se realiza de forma adecuada para dos rangos de frecuencias diferentes es decir dos tipos de suelos diferentes, y se conservan las características de los sismos registrados.

En las figuras 10 y 11, se presentan algunas variaciones paramétricas realizadas para definir la robustez del método. En la figura 10 se observa que el número de iteraciones para ajustar una señal es mayor cuando se tiene un espectro objetivo con un mayor contenido de frecuencias alto (periodos bajos), es decir cuando se ajusta un espectro para un registro en suelo rígido. Cuando se tiene un registro con contenido de frecuencias bajas (altos periodos), el ajuste requiere sustancialmente un número menor de iteraciones. Es interesante ver la figura 11, donde se observa el efecto de realizar un escalado lineal al principio del procedimiento y evitar con esto un ajuste espectral exagerado y focalizado en las HDT.

En la actualidad, existen dos controversias respecto a este método. La primera se refiere al hecho que el método genera HDT totalmente ajustadas a un espectro objetivo (i.e. función del nivel de ajuste elegido, típicamente 5\%) y debido a que por definición un espectro puede representar a más de una historia de tiempo a la vez, se podría considerar que tales HDT sintéticas sobreestiman la respuesta del sistema analizado (tanto estructural como geotécnico); La segunda se relaciona con la creencia que una HDT totalmente ajustada a un espectro de respuesta suavizado no es realista cuando éste último es comparado con espectros obtenidos de registros empíricos. 

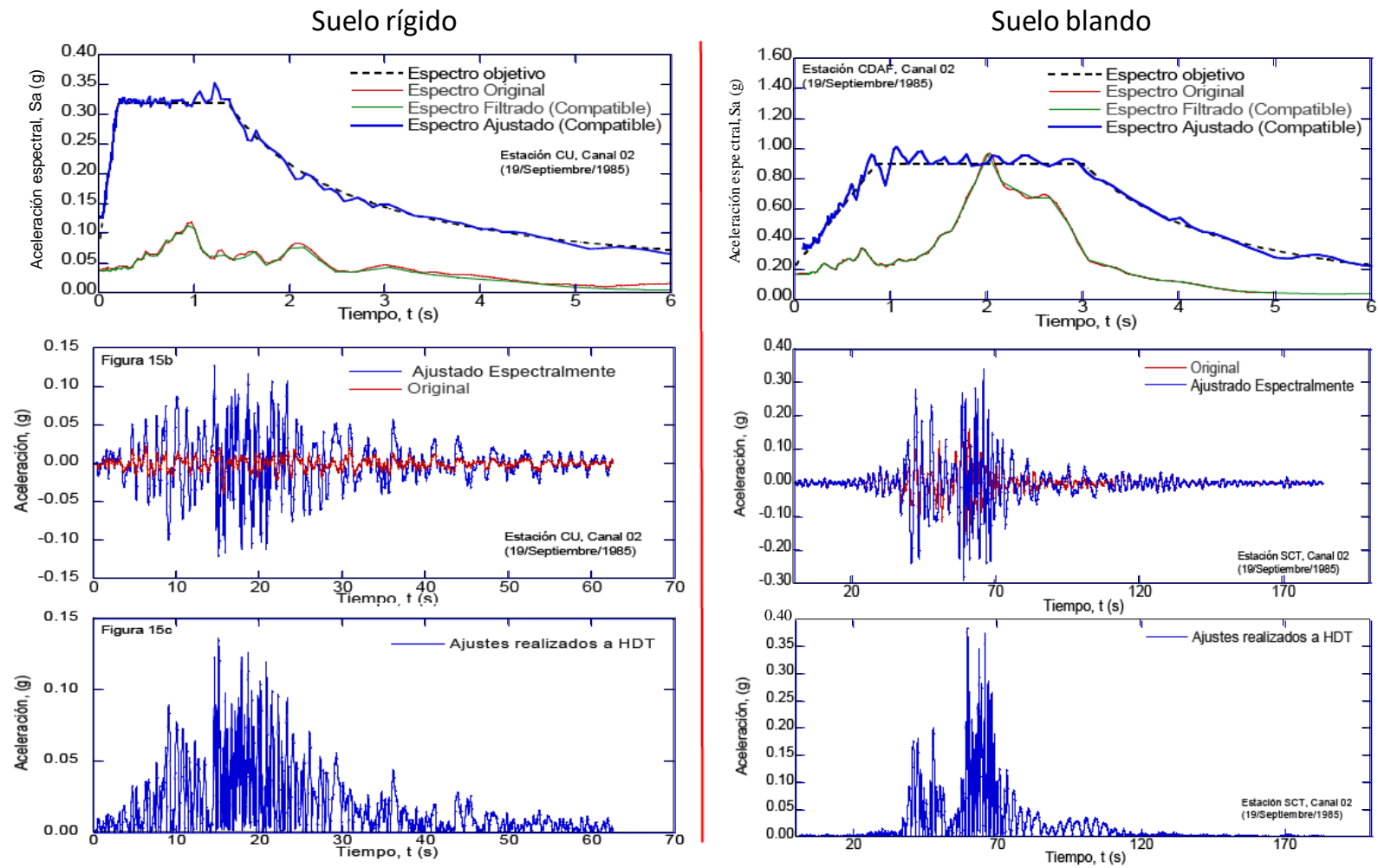

Figura 9. (a) Ajuste espectral de una HDT en suelo rígido (columna izquierda) y suelo blando (columna derecha), (b) Historias de tiempos semilla y modificada y (c) Amplitudes adicionadas a la HDT ajustada
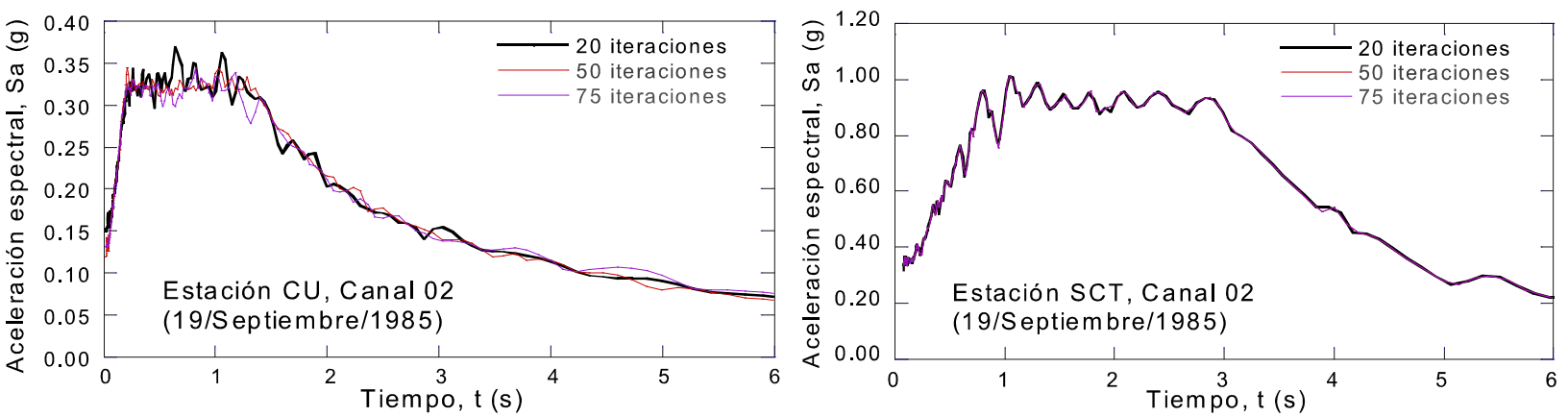

Figura 10. Variación espectral debido al número de iteraciones en función del tipo de depósito (izquierdo suelo rígido, derecha suelo blando)

\section{Generación de acelerogramas sintéticos compatibles con diversos espectros objetivo reglamentarios}

Con la finalidad de revisar la aplicación del esquema empleando wavelet, se generó un conjunto de análisis para definir acelerogramas sintéticos cuyos sismos semillas (cuatro registros sísmicos) y espectros objetivo se enlistan en la tabla 1. Como se observa en el siguiente conjunto de análisis, es necesario como primera actividad generar el filtrado de la señal original utilizando frecuencias de corte apropiadas con lo cual se obtendrá una HDT (figuras 12c a 15c) y espectros filtrados compatibles (figuras 12a a 15a); como se observa en las figuras $12 \mathrm{e}$ a $15 \mathrm{e}$, únicamente es necesario una pequeña adición/modificación focalizada en la HDT para obtener ajustes espectrales en el dominio del tiempo. Es necesario recordar que una vez ajustado espectralmente el sismo semilla compatible, el registro pierde tal compatibilidad, por lo que para recuperarlo es necesario generar un nuevo filtrado con las frecuencias de corte anteriormente elegidas. 

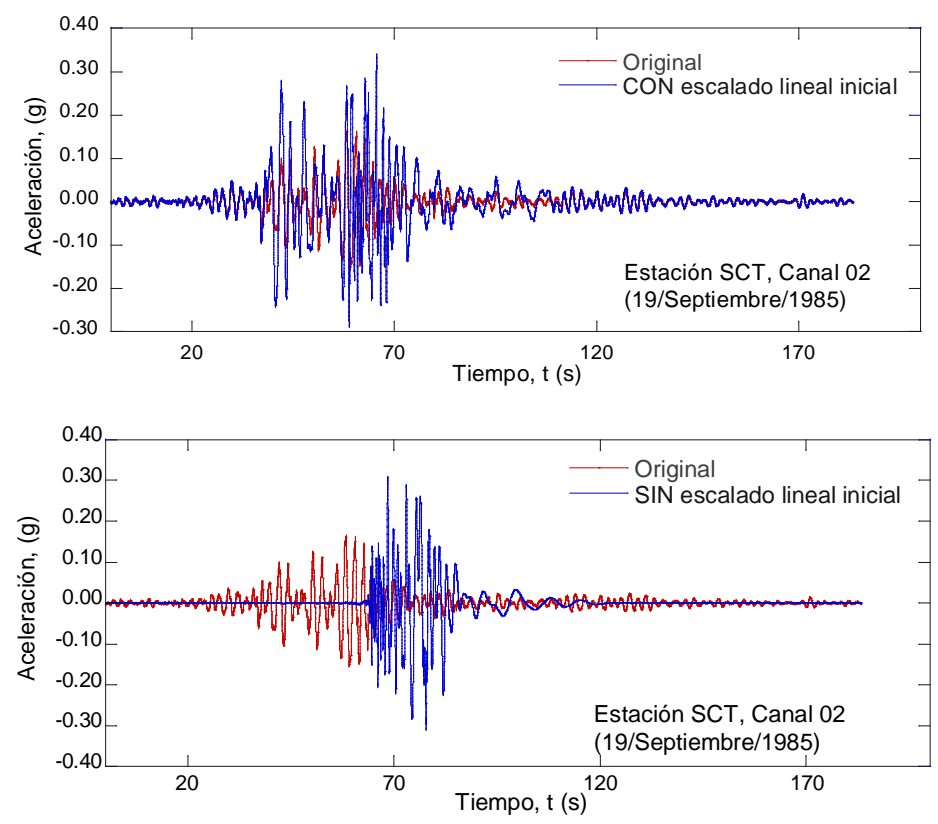

Figura 11. Efecto de utilizar un escalado lineal al inicio del procedimiento de ajuste espectral

Tabla 1. HDT semillas y espectros objetivos utilizados

\begin{tabular}{ccc}
\hline $\begin{array}{c}\text { Estación origen de la HDT semilla } \\
\text { utilizada }\end{array}$ & $\begin{array}{c}\text { Origen del Espectro objetivo } \\
\text { utilizado (Transparentes) }\end{array}$ & Figura \\
\hline Caleta de Campo (CC) & Prodisis (Manual CFE, 2008) & 12 \\
\hline $\begin{array}{c}\text { Ciudad Universitaria (CU) } \\
\text { Central de Abastos Frigorífico (CDAF) }\end{array}$ & & 13 \\
\cline { 1 - 1 } $\begin{array}{c}\text { Secretaría de Comunicaciones y } \\
\text { Transportes (SCT) }\end{array}$ & & 14 \\
\hline
\end{tabular}

Utilizando las recomendaciones anteriormente presentadas, se obtiene una HDT ajustada espectralmente y compatible en sus vertientes de aceleraciones, velocidades y desplazamientos (figuras $12 \mathrm{~b}$, $\mathrm{d}$, f a 15b, d, f); es posible observar que los espectros de Fourier (figuras $12 \mathrm{~g}$ a $15 \mathrm{~g}$ ) se han modificado mínimamente.

Con el fin de comparar el contenido energético existente en el registro empírico obtenido de las distintas estaciones acelerográficas, se empleó como medición de la energía a la Intensidad de Arias (Arias, 1970), que en términos generales se puede decir que es una medida de la capacidad de daño de la energía de una historia de tiempo. Como se aprecia en las figuras $12 \mathrm{~h}$ a $15 \mathrm{~h}$, la modificación existente aún después del ajuste espectral en el dominio del tiempo es relativamente pequeña y se considera aceptable en términos de contenido energético. 

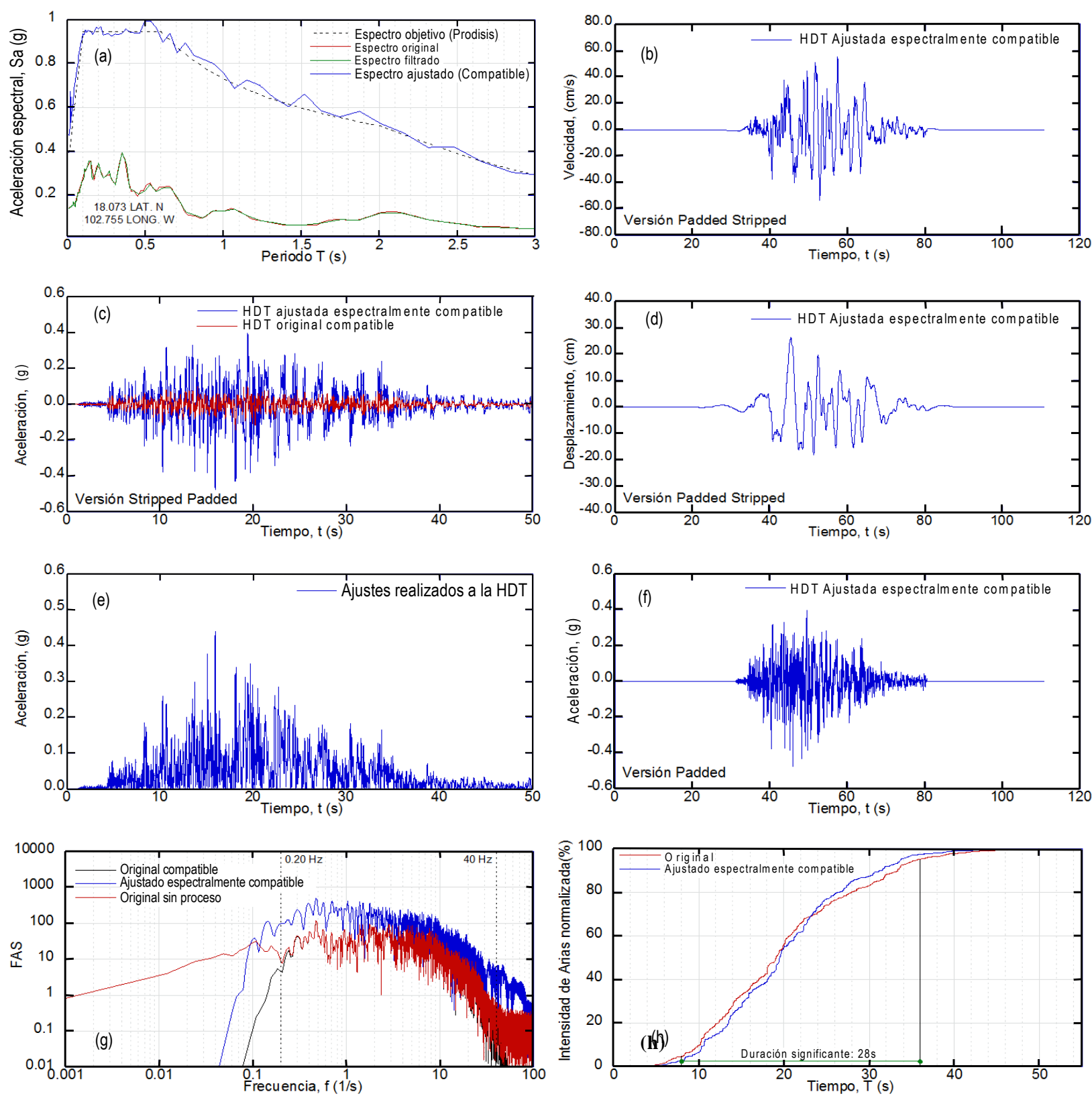

Figura 12. Resultados obtenidos al emplear el procedimiento propuesto para el filtrado y ajuste espectral en un registro de la estación CC (Michoacán, México) para el evento sísmico del 19/09/1985 (Ms=8.1) 

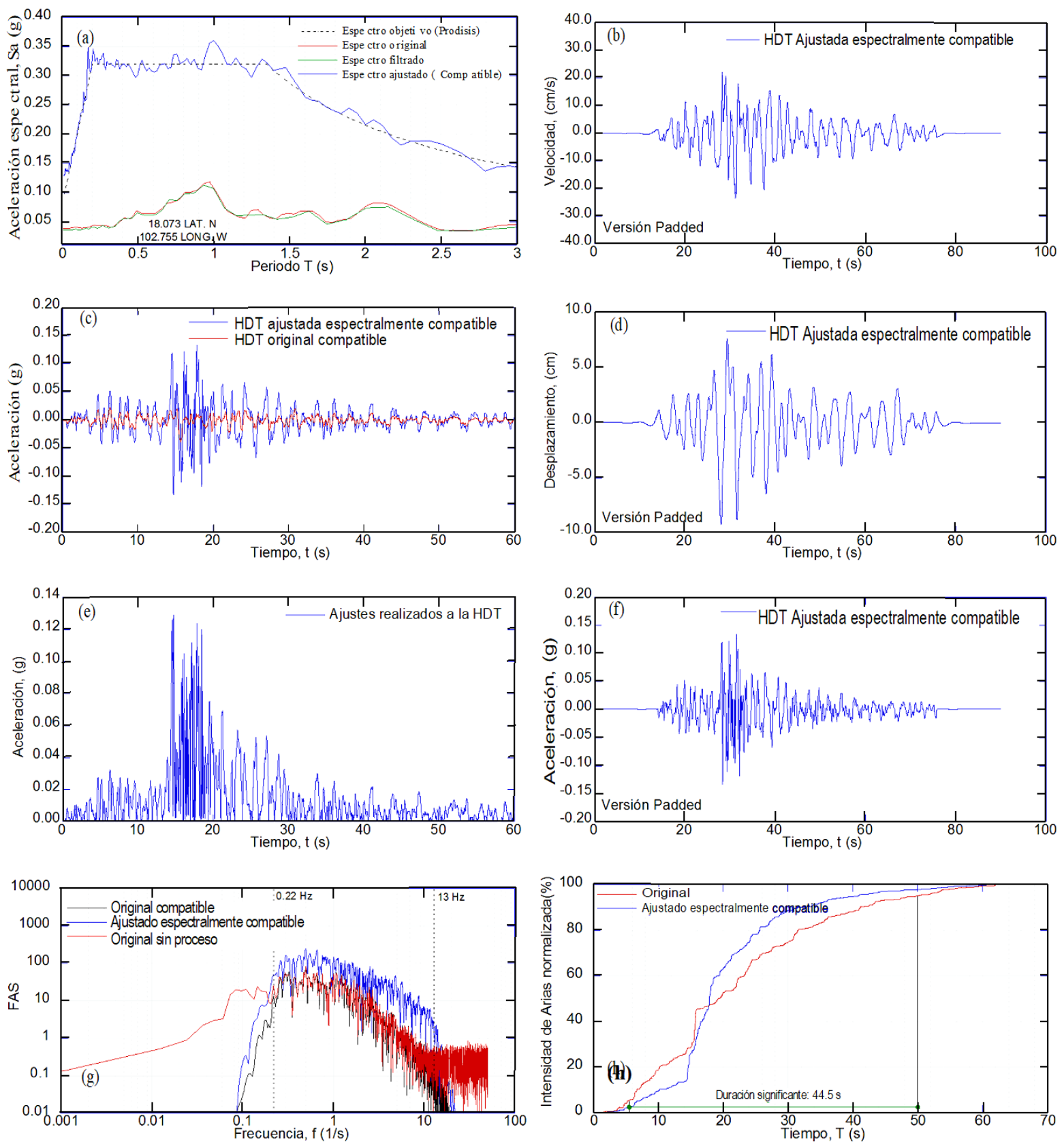

Figura 13. Resultados obtenidos al emplear el procedimiento propuesto para el filtrado y ajuste espectral en un registro de la estación CU (DF, México) para el evento sísmico del 19/09/1985 (Ms=8.1) 

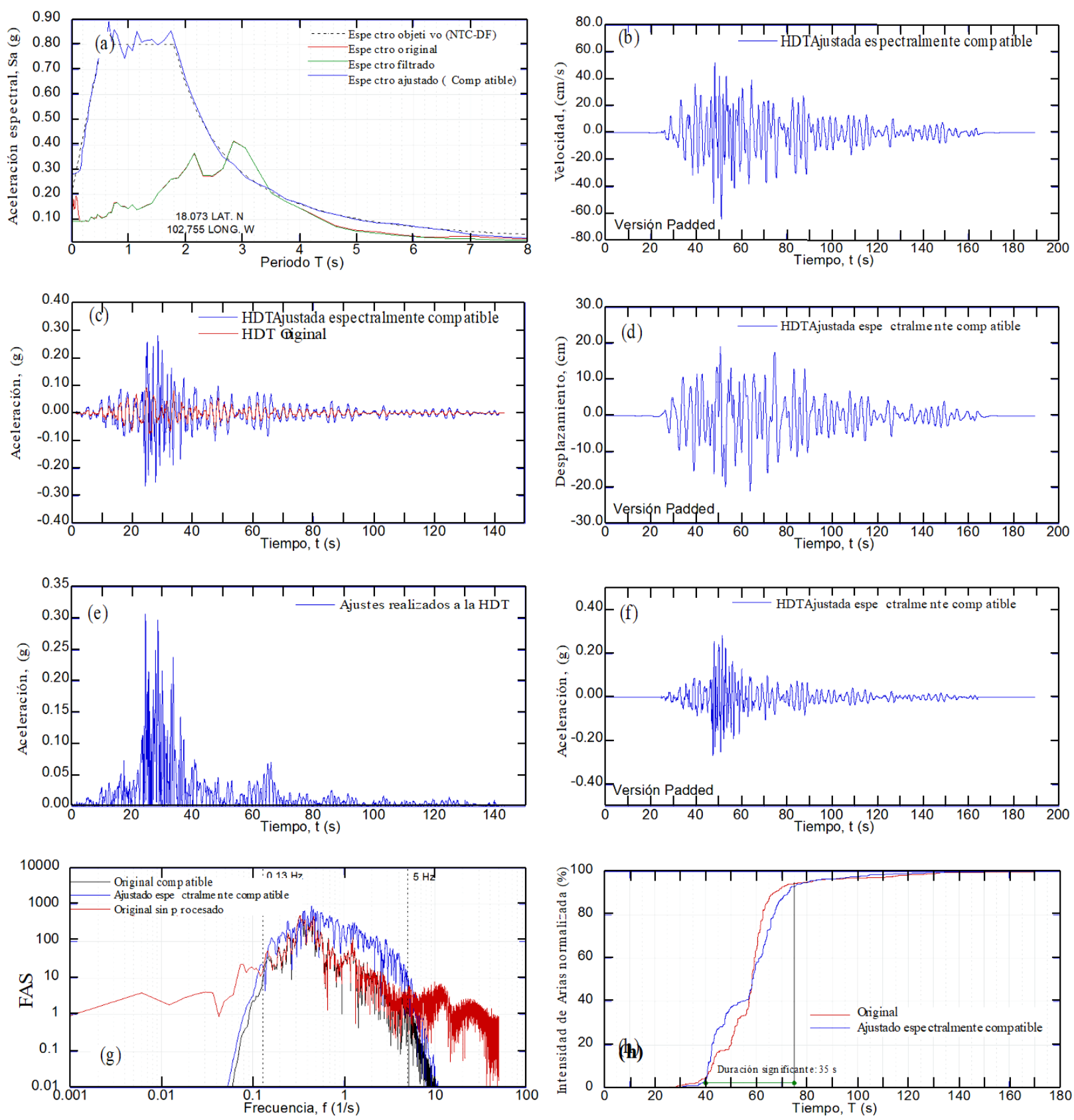

Figura 14. Resultados obtenidos al emplear el procedimiento propuesto para el filtrado y ajuste espectral en un registro de la estación SCT (DF, México) para el evento sísmico del 19/09/1985 (Ms=8.1) 

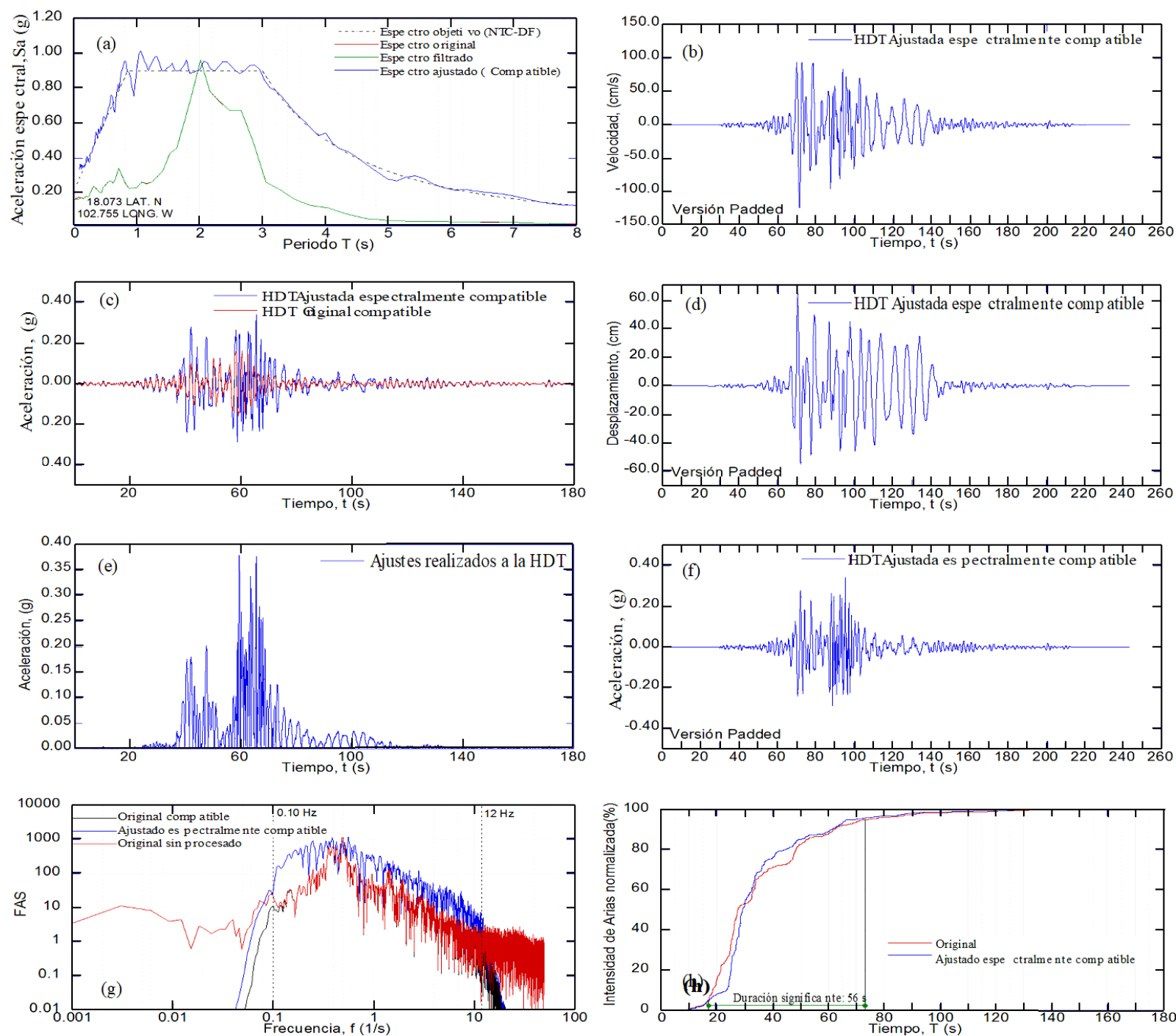

Figura 15. Resultados obtenidos al emplear el procedimiento propuesto para el filtrado y ajuste espectral en un registro de la estación CDAF (DF, México) para el evento sísmico del 19/09/1985 (Ms=8.1)

\section{Generación de acelerogramas sintéticos compatibles con diversos espectros deterministas y probabilistas}

Para describir el ambiente sísmico en una zona en estudio empleando el método determinista, se identifican las estaciones sismológicas en roca y/o terreno firme que se encuentran más cercanas al área a analizar. Con la finalidad de ejemplificar la generación de acelerogramas sintéticos basados en espectros de respuesta envolventes, se analizaron los registros obtenidos de las estaciones sismológicas correspondientes a Ciudad Universitaria (CU01, SXCU, CUMV y CUP5), el Centro Nacional de Prevención de Desastres (CENA) y el Observatorio de Tacubaya (TACY).

Una vez seleccionadas las estaciones en terreno firme se consideran los registros de sismos que tuvieran una magnitud de momento mayor que 6.5 (44 registros seleccionados). La información de los registros sísmicos fue obtenida de la Base Mexicana de Datos de Sismos Fuertes de México, BMDSF, 1996 y de la Base de Datos de Registros Acelerográficos de la Red Sísmica Mexicana, del Instituto de Ingeniería 
de la UNAM (Pérez et al., 2014). De estos registros se consideraron las dos componentes horizontales. Posteriormente, se obtuvieron los espectros de respuesta de estas señales, los cuales se normalizaron con respecto a su correspondiente aceleración máxima del terreno, PGA. Los espectros normalizados fueron escalados por un valor de PGA $0.04 \mathrm{~g}$. En la figura 16a, se presenta los espectros escalados, así como, la envolvente, la media, $\mu$, y la media más una desviación estándar, $\mu+\sigma$, de estos espectros.

Por otro lado, para definir el ambiente sísmico empleado el método probabilista, se hace uso del espectro de peligro uniforme. En la figura 16b se presenta el espectro en roca propuesto por Ordaz y Reyes (1999) que corresponde a la zona de lomas de la Ciudad de México. En la misma figura se compara con el espectro de la media más una desviación estándar, $\mu+\sigma$, que corresponde al método determinista.

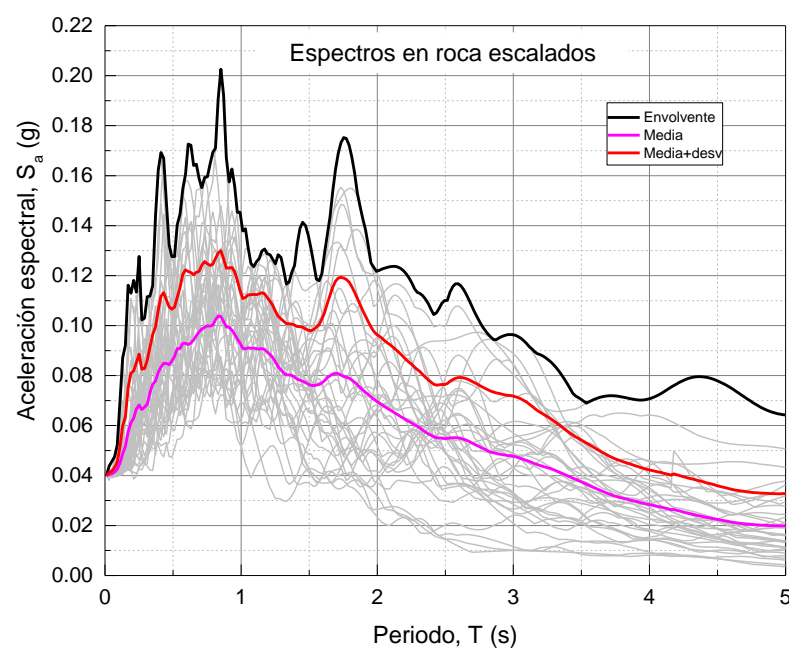

(a)

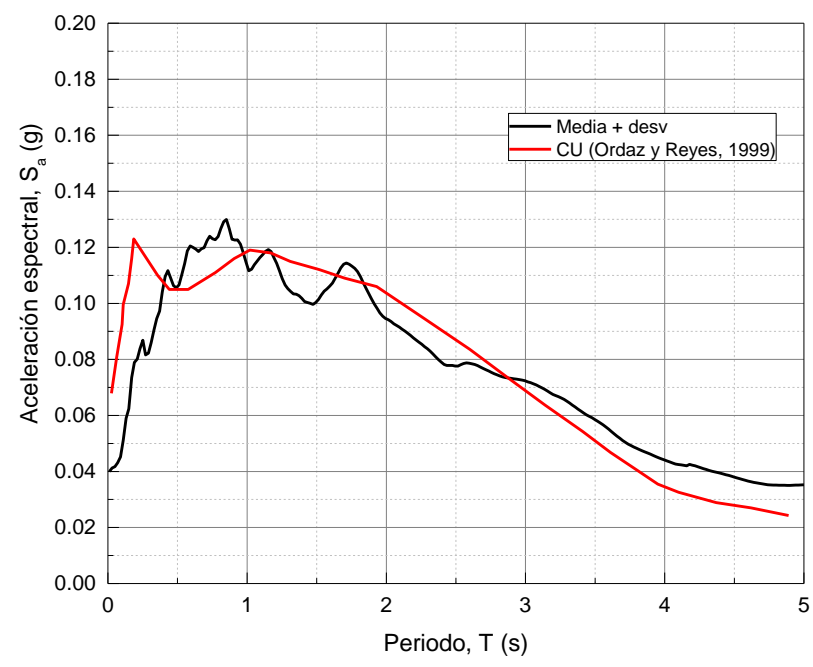

(b)

Figura 16. a) Espectros de respuesta escalados, la envolvente, la media, $\mu$, y la media más una desviación estándar, $\mu+\sigma$, de estos espectros y b) espectro de peligro uniforme (Ordaz y Reyes, 1999) y espectro de la media más una desviación estándar, $\mu+\sigma$

Con la información de los espectros de respuesta determinista y probabilista se generaron acelerogramas sintéticos. El espectro de referencia que se empleó en el método determinista corresponde a la media más una desviación estándar, $\mu+\sigma$, que resulta de los registros medidos en las estaciones sismológicas mencionadas y para el método probabilista, el espectro de referencia propuesto por Ordaz y Reyes (1999). Como acelerogramas semillas se emplearon cinco registros de aceleraciones provenientes de diferentes fuentes sísmicas, y que fueron medidos en las estaciones antes mencionadas. Las características de los sismos semilla se indican en la tabla 2 .

Tabla 2. Registros empleados para determinar los acelerogramas sintéticos para los ambientes sísmicos determinista y probabilista

\begin{tabular}{ccccc}
\hline Sitio & Evento & Ms/M & Tipo de suelo & PGA (gal) \\
\hline CENA & 15 de junio de 1999 & 6.5 & Roca & N00E $=-6.19 ;$ N90E $=-6.47$ \\
CU01 & 19 de septiembre de 1985 & 8.1 & Roca & N90E $=-32.54 ;$ N00E $=26.80$ \\
CUP5 & 20 de marzo de 2012 & 7.4 & Roca & N90E $=13.98 ;$ N00W $=11.92$ \\
CUP5 & 18 de abril de 2014 & 7.2 & Roca & N90W $=-14.87 ;$ N00W $=-12.59$ \\
TACY & 30 de septiembre de 1999 & 7.5 & Suelo duro & N90E $=9.39 ;$ N00E $=9.12$ \\
\hline
\end{tabular}


En la figura 17a se presentan los acelerogramas sintéticos generados a partir de los sismos semilla provenientes de las diferentes fuentes sísmicas, empleando como espectro de referencia la media más una desviación estándar, $\mu+\sigma$, de los espectros de respuesta escalados (método determinista). En la figura 17b se presentan los acelerogramas sintéticos generados a partir de los sismos semilla provenientes de las diferentes fuentes sísmicas, empleando como espectro de referencia el espectro de peligro uniforme propuesto por Ordaz y Reyes (1999) (método probabilista). En ambos casos se observa que la generación de los acelerogramas sintéticos es consistente para cada sismo tanto de los espectros objetivos determinista y probabilista. Para el caso de los sismos obtenidos del espectro probabilista se tiene un valor máximo mayor $(\mathrm{PGA}=0.068 \mathrm{~g})$ que el valor del PGA $(0.04 \mathrm{~g})$ del caso determinista, de ahí que las aceleraciones de las gráficas de la figura $17 \mathrm{~b}$ sean mayores que las de la figura 17a, pero la forma de los sismos es muy similar.
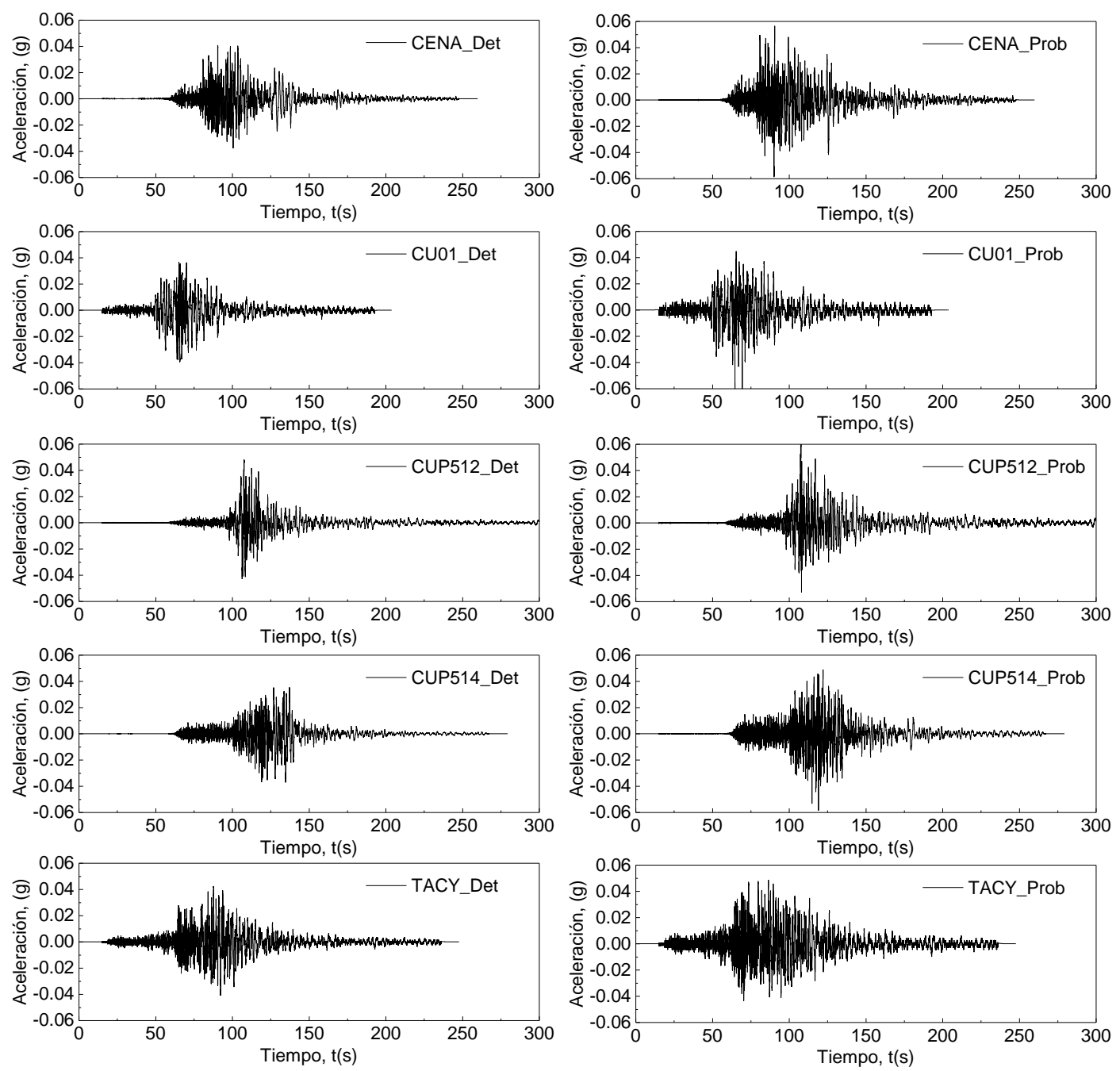

(a)

(b)

Figura 17. Acelerogramas sintéticos obtenidos para los espectros (a) determinista y (b) probabilista

Los espectros de respuesta asociados a los acelerogramas sintéticos generados se presentan en la figura 18. Con esto se muestra que la generación de los acelerogramas sintéticos empleando wavelet es muy estable y partiendo de los mismos sismos semilla se pueden generar acelerogramas sintéticos para diferentes espectros objetivos sin distorsionar o deformar la forma original de los registros sísmicos. El uso de acelerogramas sintéticos será cada vez más frecuente en la práctica de la ingeniería cuando los diseños 
geotécnicos -estructurales ante sismos se realicen con enfoques basados en desempeño (Performance based Seismic Design).

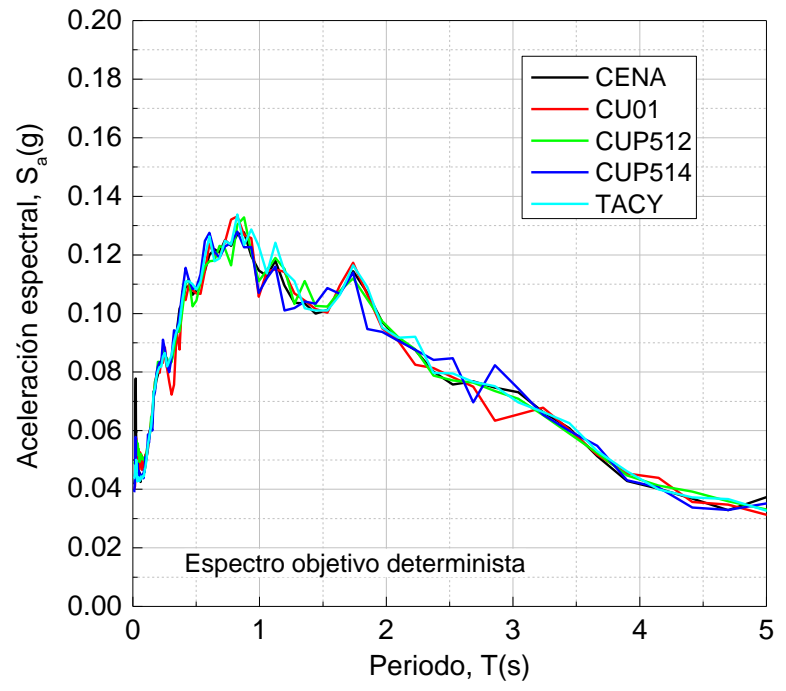

(a)

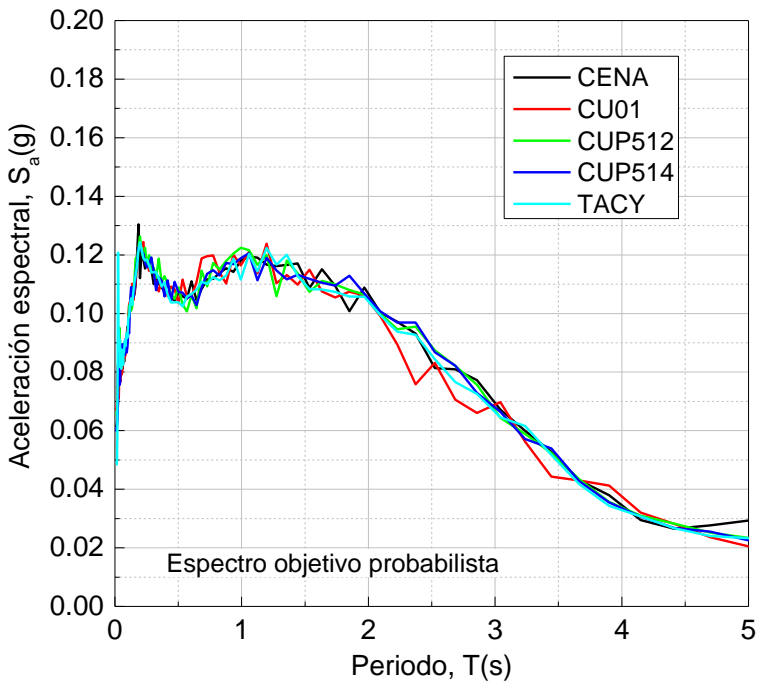

(b)

Figura 18. Espectros de respuesta asociados a los acelerogramas sintéticos obtenidos para los espectros objetivos (a) determinista y (b) probabilista

\section{ANÁLISIS DE LOS ACELEROGRAMAS MEDIANTE LA WAVELET MORLET}

El análisis mediante Wavelet es hoy en día una de las herramientas más potentes para el procesamiento de señales y ha sido empleado en diversos campos de la ingeniería (Spanos et al., 2015). En esta sección se emplea la Transforma Wavelet Continua, para analizar las características de la distribución de energía en tiempo-frecuencia de las señales registradas y los acelerogramas sintéticos desarrollados en la sección anterior.

La Transformada Wavelet Continua (CWT) de una función $f(t)$, que depende de un tiempo $t$ dado, se define como el producto interno entre la función y la familia de wavelet, $\psi$, que produce los coeficientes wavelet $C$ (Bogiatzis and Ishii, 2015). La expresión (2) muestra la CWT de una función:

$$
C(\lambda, \tau)=\left\langle\psi_{\lambda, \tau} \mid f\right\rangle=\int_{-\infty}^{\infty} f(t) \psi_{\lambda \tau}^{*}{ }_{\lambda, \tau}(t) d t
$$

donde, $\psi_{\lambda \tau}{ }_{\lambda, \tau}^{*}(t) \equiv \frac{1}{\sqrt{\lambda}} \psi\left(\frac{t-\tau}{\lambda}\right)$ denota la función wavelet, comúnmente conocida como wavelet madre, y el símbolo * indica el operador de la conjugada compleja. El parámetro $\lambda$ representa el factor de escalado, y este es un número real positivo que controla la dilatación $(\lambda>1)$ o contracción $(\lambda<1)$ de la función en el tiempo. El parámetro $\tau$ controla la traslación en el tiempo.

En este trabajo se emplea la Transformada Wavelet Continua Morlet para cuantificar los efectos de las modificaciones obtenidas en el acelerograma sintético con respecto al movimiento registrado semilla, de manera similar al procedimiento propuesto por Zhang et al., (2015). La CWT Morlet en el tiempo se define como: 


$$
C_{M}(\lambda, \tau)=|\tau|^{-\frac{1}{2}} \int_{\Delta \infty}^{\infty} f(t) \psi\left(\frac{t-\tau}{\lambda}\right) d t
$$

En la ecuación 2, $\psi(t)$ es la función wavelet Morlet, y los parámetros $\lambda, \tau$, ya fueron definidos previamente. Por lo tanto, la Transformada Wavelet Continua Morlet, puede describir la distribución de energía de la señal de tiempo $x(t)$ en el dominio conjunto tiempo-frecuencia.

Se aplicó la CWT Morlet a la señal original de CENA del 15 de junio de 1999 y a los acelerogramas sintéticos asociados a los espectros objetivos determinista y probabilista (ver figuras 17 y 18 ) y se obtuvieron los escalogramas de la figura 19, donde se puede observar la distribución de energía de las señales analizadas, los coeficientes wavelet máximos con el tiempo y los coeficientes máximos para cada periodo.
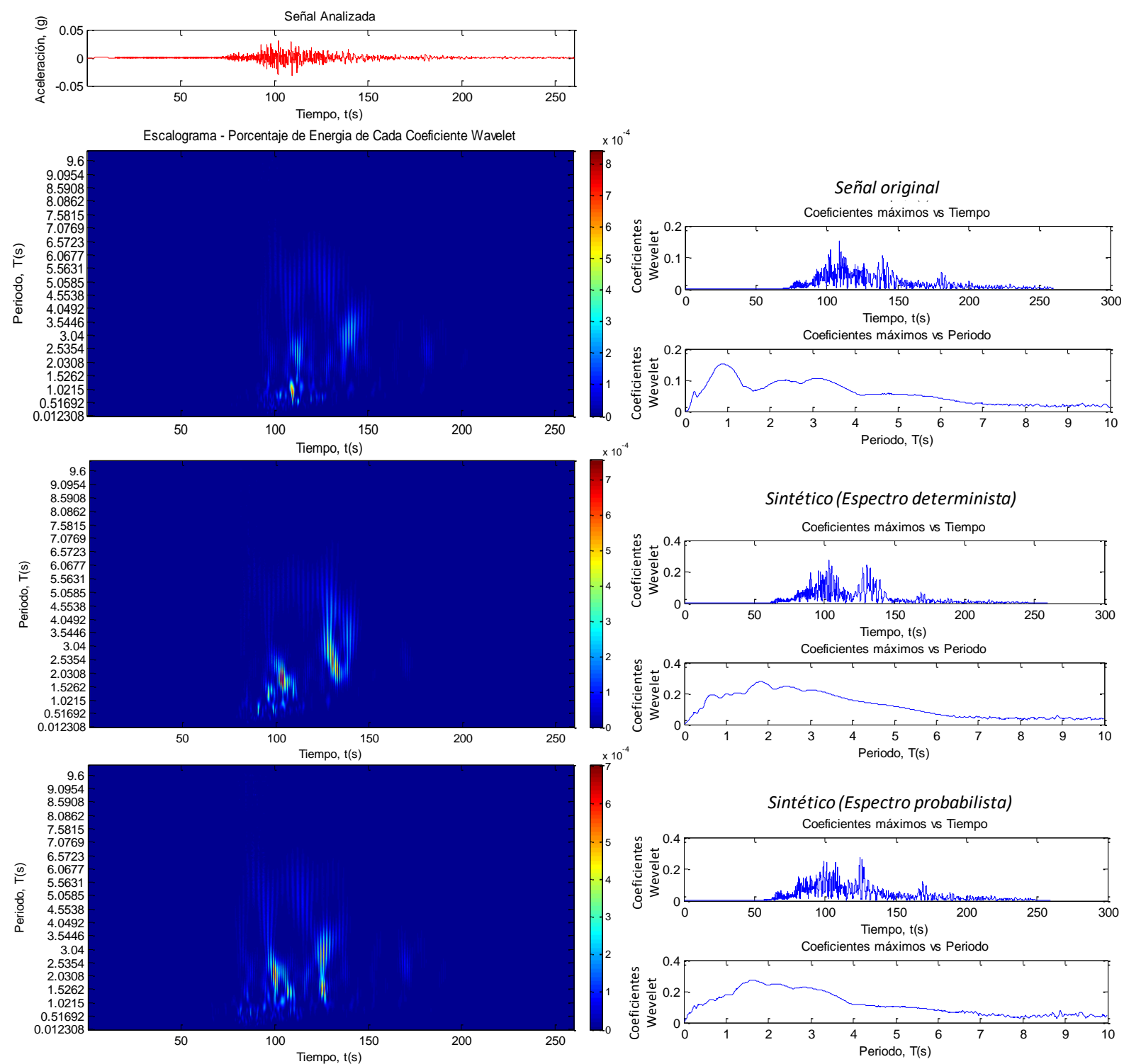

Figura 19. Escalogramas del sismo semilla, y los sintéticos asociados al espectro determinista y al espectro probabilista, los coeficientes wavelet máximos con el tiempo y los coeficientes máximos vs periodos 
En las tres señales analizadas, se observa que la mayor energía se concentra entre los 80 y los 145 segundos del sismo, lo cual indica que la metodología para modificar las señales mediante wavelet se enfoca adecuadamente en la sección de la señal con mayor energía y en las aceleraciones espectrales para los periodos que se necesitan modificar y así conservar la forma y energías de los registros sísmicos semilla. La característica principal de las wavelets para representar las señales en el dominio conjunto tiempofrecuencia parece ser muy adecuada para focalizar las modificaciones que se requieren al generar acelerogramas sintéticos a partir de mediciones de estaciones acelerográficas.

El uso de wavelets no solamente es útil en los procedimientos presentados aquí, sino que existen autores que intentan utilizar sus propiedades de visualizar y analizar una señal en el dominio de la frecuencia como en el del tiempo (con resultados prometedores), para predecir otras características de interés geotécnica, tales como licuación de arenas (Bal et al., 2014). El uso de historias de tiempo compatibles con espectros objetivos es muy importante en el campo de la ingeniería geotécnica, sobre todo en los análisis de licuación donde se presenta el exceso de presión de poro a cada ciclo de carga y descarga durante todo el evento sísmica, siendo así, la duración del sismo un factor determinante en la generación de licuación de los suelos.

\section{CONCLUSIONES}

Actualmente existe una tendencia mundial a migrar hacia los análisis en el dominio del tiempo, es decir, en la solución explícita de la ecuación del movimiento, principalmente en el área de ingeniería estructural, lo cual también está sucediendo en la ingeniería geo-sísmica; sin embargo debido al comportamiento altamente no lineal del suelo, la solución no es únicamente resolver la ecuación del movimiento, sino que existe un conjunto de actividades inherentes a la correcta modelación y uso de los registros sísmicos para su uso en los denominados análisis paso a paso, entre tales actividades destacan el filtrado y la generación de acelerogramas sintéticos ajustados a espectros objetivos para realizar el diseño de las estructuras que involucren el comportamiento no-lineal del suelo de forma explícita, además de incluir el ambiente sísmico del espectro de diseño.

Considerando lo presentado en este trabajo relacionado al ajuste espectral en el dominio del tiempo mediante wavelets, es posible mencionar las siguientes conclusiones y comentarios:

Los ajustes espectrales en el dominio del tiempo son procedimientos relativamente sencillos de ejecutar y más aún debido a la existencia de software libre de dominio público ampliamente aceptados como procedimientos pertenecientes al estado de la práctica mundial.

Los ajustes espectrales en el dominio de la frecuencia (DF), aunque útiles, generalmente es aceptado que los mismos análisis en el dominio del tiempo, son más adecuados para análisis geotécnicos, además que los análisis en el dominio de la frecuencia proporcionan señales que generalmente no presentan la firma sísmica observada en señales empíricas para un sitio específico, perdiendo la forma del acelerograma registrado.

La información necesaria para ejecutar un ajuste espectral en el dominio del tiempo, son: Espectro objetivo, rango de periodos de interés e Historias De Tiempo (HDT), es decir datos que actualmente son ampliamente disponibles en las diferentes regiones del mundo y de fácil acceso a los ingenieros de la práctica.

Las principales variables a considerar al ejecutar un análisis de ajuste espectral son: Nivel de ajuste espectral (típicamente 5\%), tipo de wavelet utilizada, número de pasadas o iteraciones de ajustes (rango de 
periodos a ajustar en cada iteración), frecuencias de corte para cada pasada y utilizar un escalado inicial al PGA al inicio del análisis.

La característica principal de las wavelets para representar las señales en el dominio conjunto tiempofrecuencia parece ser muy adecuada para focalizar las modificaciones que se requieren al generar acelerogramas sintéticos a partir de mediciones de estaciones acelerográficas.

Aunque, el procedimiento presenta grandes ventajas sobre los procedimientos en el dominio de la frecuencia, es importante tener presente que existen críticas al mismo, tales como que al generar HDT ajustadas a un espectro pueden sobreestimar la respuesta del sistema analizado y que al ser un ajuste totalmente suavizado (a la forma del espectro objetivo) no son comparables respecto a los obtenidos de registros empíricos, pero que cubren el rango de frecuencias que pudiera presentarse en un sitio para diferentes movimientos del suelo.

\section{REFERENCIAS}

Arias A (1970), “A measure of earthquake intensity", en Hansen RJ (ed) Seismic design for nuclear power plants. MIT Press, Cambridge MA, pp. 438-483.

Aki K (1967), "Scaling law of seismic spectrum", Journal of Geophysical Research, Vol. 72, No. 4, pp. 1217-1231. DOI: 10.1029/JZ072i004p01217

Al Atik, L, y N Abrahamson (2010), "An improved method for nonstationary spectral matching", Earthquake Spectra, Vol. 26, No. 3, pp. 601-617, DOI: 10.1193/1.3459159

Ayes, J C (2016), "Sismología y su aplicación en los análisis de respuesta de sitio", Examen general de conocimientos para obtener el grado de maestría en ingeniería geotecnia, Posgrado en Ingeniería, UNAM.

Ayes, J C y F A Flores (2015), "Time-history modification and spectral matching oriented to dynamic geotechnical analysis", en Fundamentals to Applications in Geotechnics, Proceedings of the 15th Pan-American Conference on Soil Mechanics and Geotechnical Engineering, 15-18 de noviembre de 2015, Buenos Aires, Argentina, IOS Press Eds., pp. 1033-1040, DOI: 10.3233/978-1-61499-603$\underline{3-1033}$.

ASCE/SEI 7-05 (2006), Minimum design loads for buildings and other structures, American Society of Civil Engineers, ISBN 0-7844-083, pp. 1-9.

BMSF (1996), Base mexicana de datos de sismos fuertes, Actualización de los catálogos de estaciones a 1995 y acelerogramas a 1994.

Bal, I, E Smyrou, P Tasiopoulou y G Gazetas (2014), "Determination of liquefaction in time domain using wavelet analysis". Proceedings of the 10th National Conference in Earthquake Engineering. DOI: 10.4231/D3JW86N60.

Bogiatzis, P y M Ishii (2015), "Continuous wavelet decomposition algorithms for automatic detection of compressional- and shear-wave arrival times", Bulletin of the Seismological Society of America, Vol. 105, No. 3, pp. 1628-1641, DOI: 10.1785/0120140267

Bommer, J y B Acevedo (2008), "The use of real earthquake accelerograms as input to dynamic analysis", Journal of Earthquake Engineering, Vol 8, No. 1, pp. 43-91. DOI:10.1080/13632460409350521

Cecini, D y A Palmeri (2015), "Spectrum-compatible accelerograms with harmonic wavelets", Computers y Structures, Vol. 147, pp. 26-35, DOI: 10.1016/j.compstruc.2014.10.013 
Comisión Federal de Electricidad, CFE (2008), Manual de diseño de obras civiles. Diseño por sismo, México.

Comisión Federal de Electricidad, CFE (2015), Manual de diseño de obras civiles. Capítulo C.1.3 Diseño por sismo, México.

Converse, M y G Brady (1992), BAP basic strong-motion accelerogram processing software, v.1.0 Department of the Interior, Geological Survey, Open-File Report 92-296-A.

Federal Emergency Management Agency, FEMA P-58-1 (2012), Seismic performance assessment of buldings, volume 1-Methodology, septiembre de 2012.

Gasparini, D y E Vanmarcke (1979), Simulated earthquake motions compatible with prescribed response spectra. Cambridge, Massachusetts: Evaluation of Seismic Safety of Buildings Report No. 2, Department of Civil Engineering, MIT.

Giaralis, A y T Spanos (2009), "Wavelet-based response spectrum compatible synthesis of accelerogramsEurocode application (EC8)", Soil Dynamics and Earthquake Engineering, Vol. 29, No. 1, pp. 219235, DOI: $10.1016 /$ j.soildyn.2007.12.002

Hancock, J, J Watson-Lamprey, N Abrahamson, J Bommer, A Markatis, E McCoy y R Mendis (2006), “An improved method of matching response spectra of recorded earthquake ground motion using wavelets", Journal of Earthquake Engineering, Vol. 10, pp. 67-89, DOI: $\underline{10.1080 / 13632460609350629}$

Hartzell, S H (1978), "Earthquake aftershocks as Green's functions”, Geophysical Research Letters, Vol. 5, No. 1, pp. 1-4, DOI: 10.1029/GL005i001p00001

Kaul, M K (1978), "Spectrum consistent time-history generation", Journal of Engineering Mechanics ASCE, Vol. 104, No. 4, pp. 781-788.

Kohrs-Sansorny, C, F Courboulex, M Bour y A Deschamps (2005), "A two-stage method for ground-motion simulation using stochastic summation of small earthquakes", Bulletin of the Seismological Society of America, Vol. 95, No. 4, pp. 1387-1400, DOI: 10.1785/0120040211

Kottke, A, X Wang y E Rathje (2013), Technical Manual for Strata. Texas: Department of Civil, Architectural and Environmental Engineering, University of Texas.

Lilhanand, K, y W S Tseng (1988), "Development and application of realistic earthquake time histories compatible with multiple-damping design spectra", Proceedings of Ninth World Conference on Earthquake Engineering, Vol. II, pp. 819-824.

Naumoski N (2001), Program SYNTH - Generation of artificial accelerograms compatible with target spectrum. Dept. of Civil Engineering, University of Ottawa, Ontario, 18.

NTCS-04 (2004), Normas Técnicas Complementarias para Diseño por Sismo, Reglamento de Construcciones para el Distrito Federal. Gaceta Oficial del Distrito Federal.

Ordaz, M y C Reyes (1999), "Earthquake hazard in Mexico City: Observations versus computations", Bulletin of the Seismic Society of America, Vol. 89, No. 5, pp. 1379-1383.

Ordaz, M, J Arboleda y S K Singh (1995), “A scheme of random summation of an empirical Green's function to estimate ground motions from future large earthquakes", Bulletin of the Seismological Society of America, Vol. 85, No. 6, pp. 1635-1647.

Pérez-Yáñez C, L Ramírez-Guzmán, A L Ruiz, R Delgado, M A Macias, H Sandoval, L Alcantara y A Quiroz (2014), "Strong ground motion database system for the mexican seismic network", American Geophysical Union, Fall Meeting 2014, San Francisco, CA, diciembre, pp. 14-19. 
RSCTH: software program for generating artificial earthquake data. http://civil.eng.buffalo.edu/engseislab/products.htm, Engineering Seismology Laboratory, State University and Buffalo, New York

Sabbetta, F y A Pugliese (1996), "Estimation of response spectra and simulation of nonstationary ground motions". Bulletin of the Seismological Society of America, Vol. 86. No. 2, pp. 337 - 352, April.

Silva, W J, y K Lee (1987), “WES RASCAL Code for Synthetizing Earthquake Ground Motions”, en: State of the Art for Assessing Earthquake Hazards in the United States, Artículo S-73-1, Reporte 24, Department of the, US Army Corps of Engineers.

Spanos P D, F Kong F, J Li y I A Kougioumtzoglou (2015), "Harmonic wavelets based excitation-response relationships for linear systems: A critical perspective", Probabilistic Engineering Mechanics, DOI: $\underline{10.1016 / j . p r o b e n g m e c h .2015 .09 .021}$

Zhang, Y, F Zhao y C. Yang (2015), "Generation of nonstationary ground motions compatible with multidamping response spectra". Bulletin of the Seismological Society of America, Vol. 105. No. 1, pp. 341 - 353, DOI: $10.1785 / 0120140038$ 